\title{
Stellar Chromospheric Activity
}

\author{
Jeffrey C. Hall \\ Lowell Observatory \\ 1400 West Mars Hill Road \\ Flagstaff, AZ 86001 \\ email: jch@lowell.edu \\ http://www. lowell.edu/users/jch
}

Accepted on 29 January 2008

Published on 10 March 2008

\begin{abstract}
The Sun, stars similar to it, and many rather dissimilar to it, have chromospheres, regions classically viewed as lying above the brilliant photosphere and characterized by a positive temperature gradient and a marked departure from radiative equilibrium. Stellar chromospheres exhibit a wide range of phenomena collectively called activity, stemming largely from the time evolution of their magnetic fields and the mass flux and transfer of radiation through the complex magnetic topology and the increasingly optically thin plasma of the outer stellar atmosphere. In this review, I will (1) outline the development of our understanding of chromospheric structure from 1960 to the present, (2) discuss the major observational programs and theoretical lines of inquiry, (3) review the origin and nature of both solar and stellar chromospheric activity and its relationship to, and effect on, stellar parameters including total energy output, and (4) summarize the outstanding problems today.
\end{abstract}

This review is licensed under a Creative Commons Attribution-Non-Commercial-NoDerivs 3.0 Germany License. http://creativecommons.org/licenses/by-nc-nd/3.0/de/ 


\section{Imprint / Terms of Use}

Living Reviews in Solar Physics is a peer reviewed open access journal published by the Max Planck Institute for Solar System Research, Max-Planck-Str. 2, 37191 Katlenburg-Lindau, Germany. ISSN 1614-4961.

This review is licensed under a Creative Commons Attribution-Non-Commercial-NoDerivs 3.0 Germany License: http://creativecommons.org/licenses/by-nc-nd/3.0/de/

Because a Living Reviews article can evolve over time, we recommend to cite the article as follows:

Jeffrey C. Hall,

"Stellar Chromospheric Activity",

Living Rev. Solar Phys., 5, (2008), 2. [Online Article]: cited [<date $>$ ], http://www.livingreviews.org/lrsp-2008-2

The date given as $<$ date $>$ then uniquely identifies the version of the article you are referring to.

\section{Article Revisions}

Living Reviews supports two different ways to keep its articles up-to-date:

Fast-track revision A fast-track revision provides the author with the opportunity to add short notices of current research results, trends and developments, or important publications to the article. A fast-track revision is refereed by the responsible subject editor. If an article has undergone a fast-track revision, a summary of changes will be listed here.

Major update A major update will include substantial changes and additions and is subject to full external refereeing. It is published with a new publication number.

For detailed documentation of an article's evolution, please refer always to the history document of the article's online version at http://www. livingreviews.org/lrsp-2008-2. 


\section{Contents}

1 Introduction $\quad 5$

2 Background and Definitions $\quad 7$

2.1 What is a chromosphere? . . . . . . . . . . . . . . . . . 7

2.2 What is activity? . . . . . . . . . . . . . . . . . . . . . . . . . . . . . . . . . . .

2.3 What stars have chromospheres? . . . . . . . . . . . . . . . . . . 10

2.4 Going further . . . . . . . . . . . . . . . . . . . . . 12

3 The Care and Feeding of a Chromosphere 13

3.1 Early theory and models of the chromosphere . . . . . . . . . . . . . . . 13

3.2 Long-term observations of Ca II H \& K . . . . . . . . . . . . . . . . . . . . . . . . . . . . . . . . .

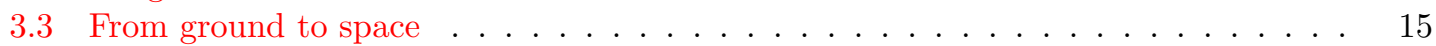

3.4 Semi-empirical models . . . . . . . . . . . . . . . . . . . . . 16

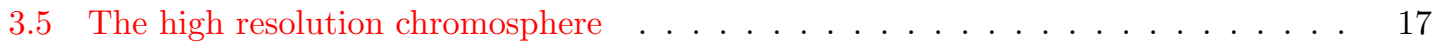

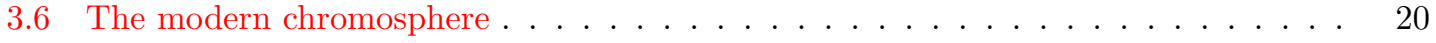

3.7 Going further . . . . . . . . . . . . . . . . . . . . . 20

4 Chromospheric Activity 2

4.1 Synoptic observations and surveys of Ca II H \& K . . . . . . . . . . . . . . . . . . . 22

4.2 Measuring chromospheric activity . . . . . . . . . . . . . . . . 25

4.3 The Vaughan-Preston Gap and the evolution of stellar dynamos . . . . . . . . . . 26

4.4 Basal heating of the chromosphere . . . . . . . . . . . . . . . . . . . . . . . . . . . . . . . . . .

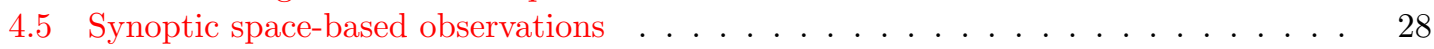

4.6 Sun-as-a-star observations . . . . . . . . . . . . . . . . . . . . . . . . 29

4.7 Chromospheric activity and brightness variations . . . . . . . . . . . . . . . . . . 29

4.8 Flat activity stars and the Maunder Minimum . . . . . . . . . . . . . . . . . 31

4.9 Going further . . . . . . . . . . . . . . . . . . . 35

$\begin{array}{llr}5 & \text { A View to a Chromosphere } & 37\end{array}$

6 Acknowledgments $\quad 39$

$\begin{array}{ll}\text { References } & 40\end{array}$ 



\section{Introduction}

This is a review of stellar chromospheric activity. I will discuss how scientists have defined this term in Section 2, but for now, let us consider the scope of the problem. The chromosphere (in the classical, stratified, and highly oversimplified view) is an intermediate region in the atmosphere of a star, lying above the photosphere and below the corona. Chromospheric activity, which encompasses diverse phenomena that produce emission in excess of that expected from a radiative equilibrium atmosphere, is tightly linked to changes in the stellar magnetic field, whether periodic or irregular, and is therefore tied to the structure of the subsurface convection zone, the star's rotation, and the regeneration of the magnetic field via a self-sustaining dynamo. Since the chromosphere is not in radiative equilibrium, non-thermal mechanisms of energy deposition must be present, and they take both magnetic and non-magnetic forms. The luminosity of the Sun and Sun-like stars is correlated with their activity levels, so chromospheric activity and phenomena associated with both affect the rarefied outer atmosphere of the star, and to some extent reflect the star's global properties.

This swarm of facts can be rearranged into a crude but somewhat more succinct picture: the structure of the chromosphere is profoundly affected by the interior structure of the star as well as its gross parameters (e.g., mass, rotation rate), and the chromosphere in turn profoundly influences the nature and variability of the emergent spectrum, particularly in the ultraviolet. Understanding chromospheric activity and variability is therefore essential to a complete understanding of the physics and evolution of a star and, by virtue of the ionizing ability of the short wavelength radiation it emits, to an understanding of the variability of the heliosphere and Earth's atmosphere.

Studies of the chromospheric activity of stars have benefited greatly from complementary studies of the Sun, to the point that a review of stellar chromospheric activity would be woefully incomplete without devoting significant space to solar studies. While the stars provide a laboratory for deducing chromospheric properties across a range of masses and ages, the Sun's proximity allows high-resolution observation of one (hopefully typical) chromosphere, which we can use as a springboard to test hypotheses about stellar chromospheres in general, and as a vital picture of what the unresolved stellar structures may look like. Therefore, although I use the terms solar and stellar to mean "Sun" and "other than Sun", I will take care not to consider solar and stellar studies in isolation.

These considerations open a number of portals into an enormous literature, so this review could be of deadly proportions. To keep things manageable, I have condensed the material into three sections, each addressing one of three broad questions.

In Section 2, I ask the deceptively simple question: What is a chromosphere? This encompasses the definition of a few essential terms.

In Section 3, I ask: What does a chromosphere look like? Here I summarize the development of chromospheric activity research, both theoretical and observational, since about 1960, with the general purpose of showing how our understanding of chromospheric structure has evolved from the classical, time- and space-averaged view to today's dynamic picture.

In Section 4, I address the question: How does a chromosphere vary with time? With an emphasis on long-term, synoptic observations of Sun-like stars, I review how activity is manifested in the chromosphere, how it evolves, and the astrophysical as well as practical implications of this variability.

Each of these sections has several subsections that deal with a particular aspect of the question under consideration, covering the historical context as well as the present state of our understanding. Throughout, I attempt to point readers to recent work as well as the fundamental references, given the relative ease today of searching both backward and forward through the literature using online resources. Anecdotes and further entry points into the literature are provided in the Going further subsections that conclude each of Sections 2, 3, and 4 . 
Finally, in Section 5, I revisit the question of what constitutes a chromosphere with the more complete perspective that the previous sections provide, and offer a few thoughts about productive lines of work in the next decade.

Per the guidelines for a Living Review, I assume throughout that the reader has at least a beginning graduate training in astrophysics, but is not as afflicted by an interest in chromospheres as the author or those cited herein. The sections hopefully are both useful summaries of the literature for stellar workers and readable introductions for non-specialists and students. (For each interesting study and result cited, I must apologize to the authors of the 20 or 30 complementary references omitted or overlooked.) If by the end one has a general idea of what a chromosphere looks like, how it varies with time, and what researchers are working on today, I have achieved the intended result. 


\section{Background and Definitions}

\subsection{What is a chromosphere?}

In this section, I present a qualitative description of the chromosphere and its characteristics, prefatory to a similarly introductory definition of activity in Section 2.2.

The outer atmosphere of the Sun is invisible in white light, washed out by the brilliant radiation from the photosphere. Awareness of the Sun's outer atmosphere developed during the 1700s and 1800s thanks to total eclipses, as observers began to take note of the extended corona, as well as the "red flames" we now call prominences and the pink ring of emission at the solar limb that we call the chromosphere (see Figure 1). These phenomena were confirmed to be of solar rather than terrestrial origin by the mid 1800s, and spectroscopic observations began with an eclipse visible in India and Malaysia in 1868. Observations were soon being made outside of eclipses as well, using prism spectrometers to image the Sun in narrow emission passbands. The early observations led promptly to the discovery in the solar spectrum of a new element, appropriately named helium, some two decades before it was discovered on Earth. Subsequent work led to the realization that spectra of both the chromosphere and the corona contain numerous emission features of hightemperature ionized species, indicating that temperature in the solar atmosphere, after dropping from $\approx 6500 \mathrm{~K}$ to $4400 \mathrm{~K}$ (and possibly down to $3800 \mathrm{~K}$ in places) through the photosphere, rises to an extended plateau at about $7000 \mathrm{~K}$ in the chromosphere, and then abruptly jumps to over $1,000,000 \mathrm{~K}$ in the corona. More fundamentally, the physical extent of the temperature rise is incompatible with thermal processes alone, so the escape of energy from the photosphere to empty space must involve mechanisms beyond simple equilibrium transfer of radiation.

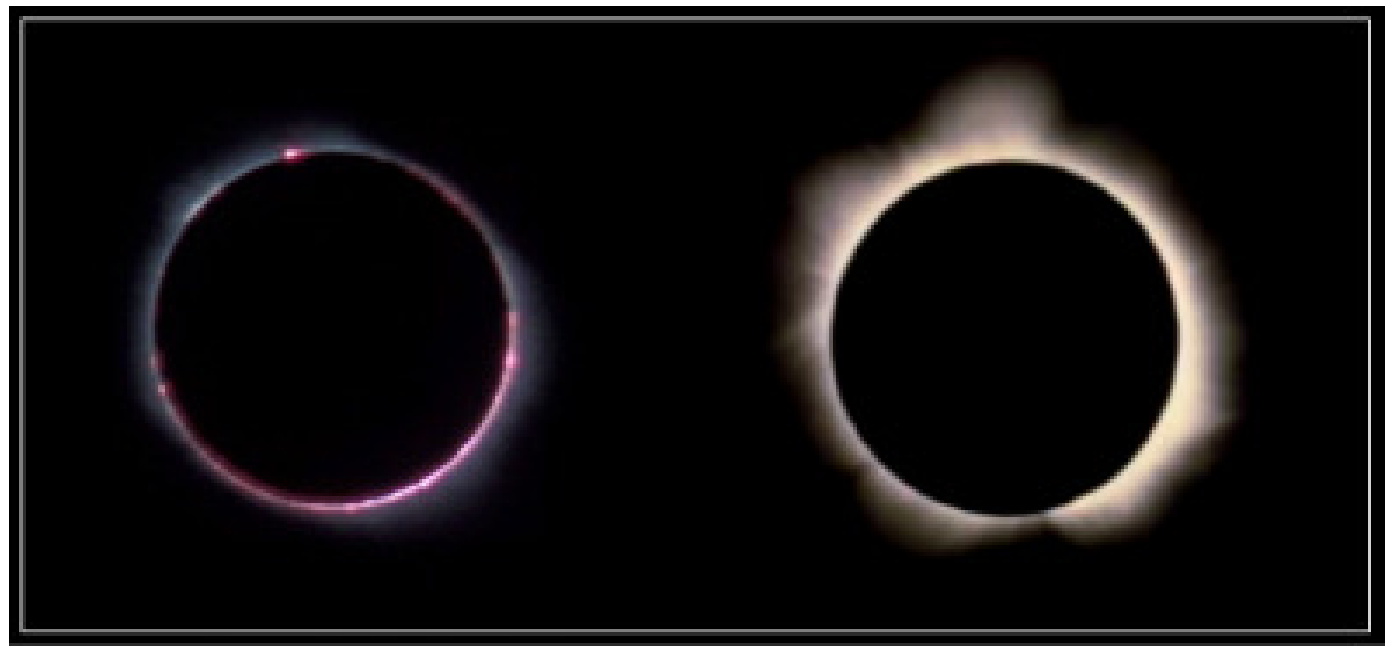

Figure 1: Two views of the Sun during a total solar eclipse. Left: the pink chromosphere is visible, its color stemming from $\mathrm{H} \alpha$ emission at $6563 \AA$ (image courtesy Dr. William Cohen; used by permission). Right: the corona is visible in scattered photospheric light (image by Bill Livingston/NSO/AURA/NSF).

The term chromo-sphere carries at least some implication that we are considering a "layer" of plasma overlying the photosphere, but it has long been known that the chromosphere is quite heterogeneous. Roberts (1945) published observations of "small spike" prominences, which he called spicules and which are both ubiquitous and evanescent features of the chromosphere, appearing and disappearing on timescales of minutes. They and their larger cousins, the macrospicules (Bohlin et al., 1975), are tightly collimated jets of plasma streaming upward through the chromosphere. Spicules are bright in $\mathrm{H} \alpha$, giving the chromosphere its pink color, while the larger and hotter 
macrospicules are also prominent in extreme ultraviolet images (see Figure 2). Alongside these bright features, however, we observe widespread and extremely cool gas at chromospheric heights, revealed by the presence of $\mathrm{CO}$ bands and picturesquely described as a chromospheric "heart of darkness" (Solanki et al., 1994). These observations, along with others I will discuss later, put to rest any notion that the chromosphere constitutes a well-defined layer; rather, it is extremely inhomogeneous, variable on short and long timescales, and characterized by strongly confined and directed regions of hot plasma that suggest a complicated magnetic topology.

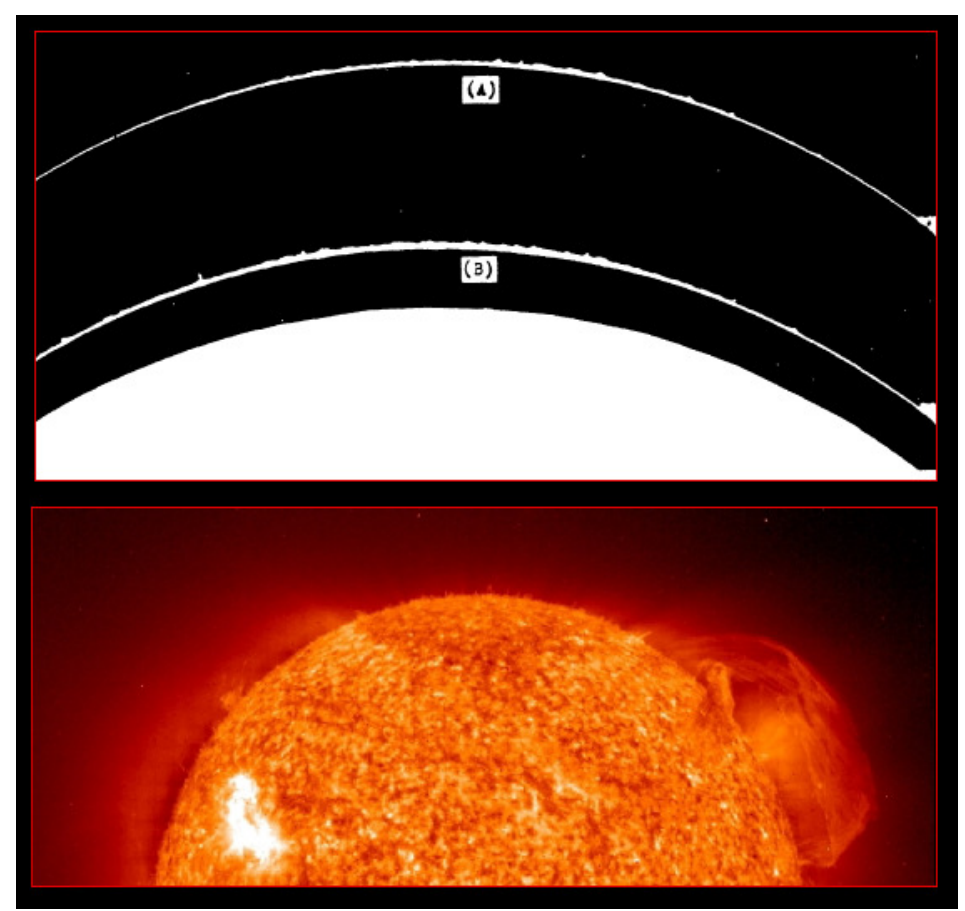

Figure 2: Top: Roberts (1945) published photographs of chromospheric spicules taken eight minutes apart (image A and B), illustrating their rapidly changing character; note especially the appearance of two well-defined spicules at left in image B (from The Astrophysical Journal). Bottom: An image of the Sun obtained by the Solar and Heliospheric Observatory (SOHO) in the light of He II at $304 \AA$. The huge prominence at right (which Huggins, Lockyer, and their 19th century contemporaries would have called a "red flame") dominates the scene, but elsewhere are numerous macrospicules, especially at top. The chromosphere is highly inhomogeneous, dynamic, and topologically complex (image courtesy SOHO/EIT; ESA and NASA).

Small wonder, then, that researchers have struggled with the apparently simple question, what is a chromosphere? Numerous definitions have been advanced, variously based on height, temperature, physical processes, or some combination thereof. Reviewing this question herein will be a circular task, since activity itself defines the chromosphere to a significant extent, but for now let us consider Figure 3, from the definitive "early" model of the solar chromosphere (Vernazza et al., 1981). Height in the atmosphere increases to the left, and the lower $x$-axis shows the column mass density.

In this model, the temperature declines through the photosphere to a height of about $500 \mathrm{~km}$, rises slowly to about $8000 \mathrm{~K}$ at $2000 \mathrm{~km}$, and then rises sharply. For now, let us take a working definition of the chromosphere to be the regions of a stellar atmosphere (a) where we observe emission in excess of that expected in radiative equilibrium and (b) where cooling occurs mainly by radiation in strong resonance lines (rather than in the continuum as is mostly the case in the

Living Reviews in Solar Physics

http://www. livingreviews . org/lrsp-2008-2 
photosphere) of abundant species such as Mg II and Ca II. If we take the naive view that this defines a homogeneous, spheroidal shell in the solar atmosphere, then Figure 3 suggests that for the Sun, the chromosphere is roughly $1700 \mathrm{~km}$ thick and reaches perhaps 25,000 K in its uppermost part. As discussed above, the picture is considerably more complex, so our definition is intentionally worded to avoid speaking of parameters like heights and temperatures, but rather of two essential chromospheric physical processes, and giving no implication that these processes occur in a spatially uniform way.

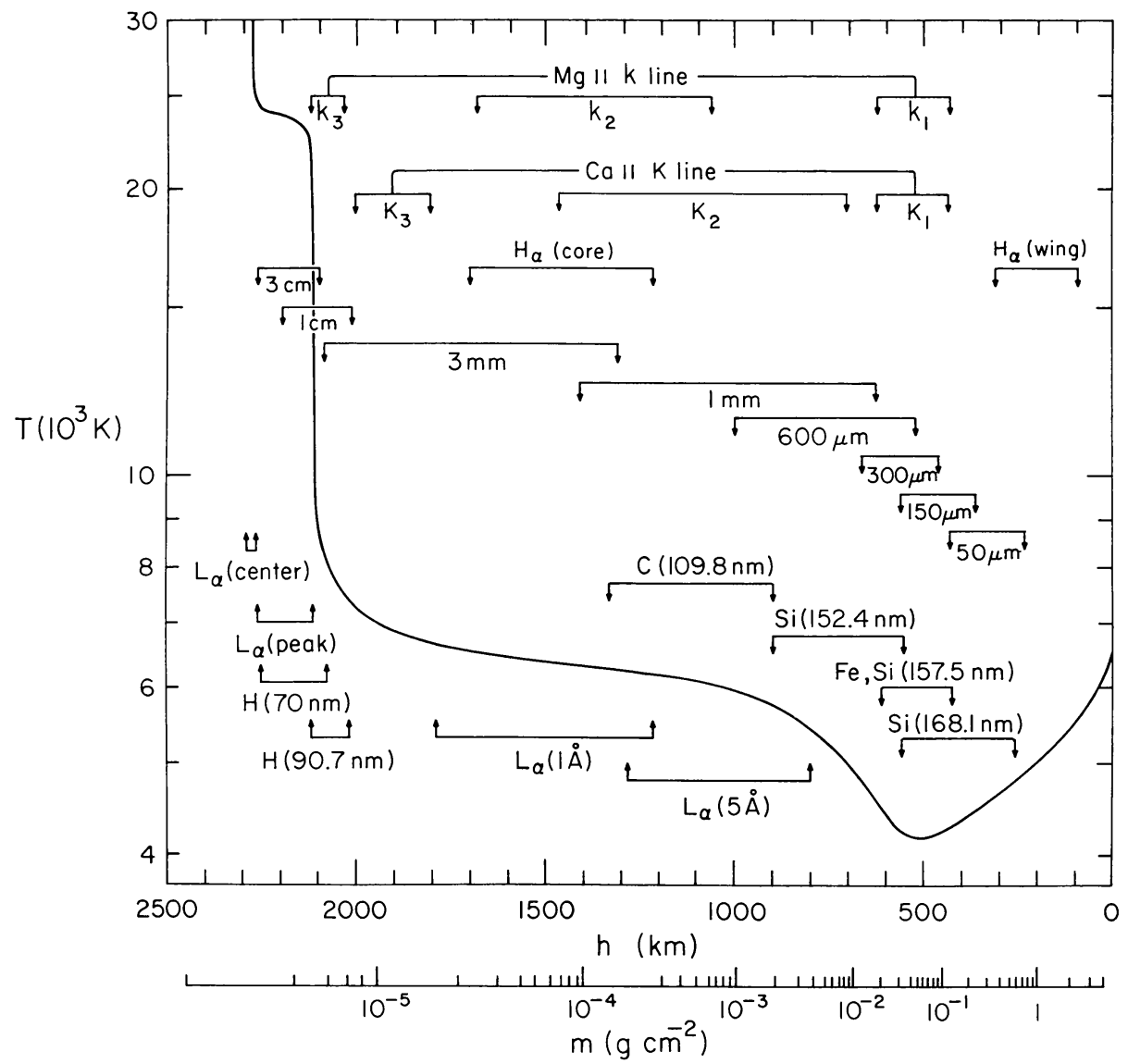

Figure 3: Figure 1 from Vernazza et al. (1981). The temperature structure derived from a semi-empirical model of the solar chromosphere is presented, along with the formation heights of important lines and continua. Very roughly, the solar chromosphere lies between the temperature minimum at right and the rapid rise toward transition region and coronal temperatures at $\approx 2300 \mathrm{~km}$. A few important points: $(1)$ heating and cooling via hydrogen is sufficiently central to chromospheric energy balance to form part of a definition of a chromosphere, (2) other than a very limited set of features such as Ca II $\mathrm{H} \& \mathrm{~K}, \mathrm{H} \alpha$, and the Ca II infrared triplet, chromospheric lines lie in the UV or beyond and are not accessible from the ground, and (3) a given point in a real chromosphere may look profoundly different from this "average" chromosphere.

\subsection{What is activity?}

Having developed at least an approximate definition of a chromosphere in Section 2.1, let us now define activity. 
In a stellar atmosphere in radiative equilibrium (RE), energy transport through the plasma is purely by radiation, and any heat absorbed from the radiation field is balanced by the thermal emission of the plasma back to the photon flux, to maintain the outward flow of energy from the deep interior. It has been known for some time that the outward temperature rise one usually associates with a chromosphere can occur under special circumstances in radiative equilibrium (e.g., Cayrel, 1963; Auer and Mihalas, 1969; Skumanich, 1970), but also that emission reversals in prominent Fraunhofer lines such as CaII H \& K are a sure sign of departures from RE; additional mechanisms of heating, generally termed activity, are required to explain the additional radiative losses in these and other lines. This activity takes two principal forms.

Babcock (1961) described a model by which a self-regenerating magnetic field could explain the principal features of visual and magnetic observations of the sunspot cycle, and since then the evolution and variability of solar and stellar magnetic fields has been found to account for much of what we observe as activity in the chromosphere and corona, via heating by Alfvén waves or transport of mechanical energy along the magnetic "conduits" into the outer atmosphere. Much of this review, therefore, will be concerned with our theoretical and observational understanding of the magnetic properties and behavior of stars.

An alternative form of activity was proposed by Biermann (1948) and Schwarzschild (1948), who discussed how the solar granulation, which occurs as myriad convective cells rise to the solar surface and release energy in the photosphere, could generate a continuous stream of acoustic waves that propagate into the outer atmosphere, heating it as they develop into shocks and dissipate. Dissipation of acoustic energy as a source of extra heating, and how it propagates into the outer stellar atmosphere, has since been widely explored.

This activity is not only central to the definition of a chromosphere, but drives its essential structure in the following qualitative way. As noted just above, in the Sun and stars like it, phenomena exist that dump mechanical energy into the atmosphere overlying the mainly neutral photosphere, causing heating beyond the expected RE values for the increasingly tenuous plasma. The plasma can balance the energetic books through a steadily increasing hydrogen ionization fraction as it warms from $\approx 5000$ to $\approx 8000 \mathrm{~K}$, which releases a large pool of electrons that allows the collisional radiative cooling that forms part (b) of our definition above. This happens over a relatively thick region, explaining the large extent of the chromospheres of Sun-like stars. Once hydrogen becomes fully ionized, however, the plasma loses this critical cooling mechanism; not surprisingly, this happens at the point near the left side of Figure 3 where the temperature rises rapidly from the chromospheric "plateau" to coronal temperatures.

\subsection{What stars have chromospheres?}

"I'll buy chromospheres for all types of stars," said R.N. Thomas (in Jordan and Avrett, 1973, p. 48), and under some definitions one may indeed argue for a chromosphere in almost any star. However, the types of activity discussed above also strongly suggest in which stars we may expect to find chromospheres in the theoretical sense given in Section 2.1; i.e., an unexpectedly thick region of the stellar atmosphere characterized by non-radiative heating and cooling occurring predominantly in resonance lines rather than the continuum.

First, we expect to find thick chromospheres primarily in cool stars due to the structural considerations given in the previous section. Dissipation of excess mechanical heating can happen in cool stars via ionization of hydrogen as the plasma warms at increasingly large heights above the photosphere; hot stars with partially or highly ionized photospheres have already "used up" this electron pool at their visible surfaces, and thus cannot support the extended chromospheres we see in the cool half of the Hertzsprung-Russell (HR) diagram. Second, both the magnetic and nonmagnetic sources of activity mentioned above imply the presence of surface convection, the former through its critical role in maintaining the magnetic dynamo via subsurface bulk mass transport,

Living Reviews in Solar Physics

http://www.livingreviews.org/lrsp-2008-2 
and the latter explicitly. In this review of chromospheric activity, therefore, I consider those stars for which a subsurface convection layer is present. This will occur in roughly in late A and cooler dwarfs, and in more massive stars as they leave the main-sequence and develop convective zones.

Observations amply support these theoretical arguments, and can be used to construct a "chromospheric HR diagram" showing rather precisely where Sun-like chromospheres are expected to be found, as well as illuminating important aspects of stellar structure and evolution.

Toward the thin convection zone limit, evidence has been found for chromospheric emission in dwarfs as hot as Altair, A7 IV-V (Freire Ferrero et al., 1995), and Simon et al. (2002) concluded from Far Ultraviolet Spectroscopic Explorer (FUSE) observations of a sample of A dwarfs that high temperature emission indicative of coronae, and by inference chromospheres, appears at about $8250 \mathrm{~K}$. The chromospheres near this limit are of course quite weak; Simon et al. (2002) found emission for these stars to be at most a few percent of solar values. However, the onset of the emission appears to be abrupt and well determined, suggesting an equally abrupt transition from radiative to convective stellar envelopes at an effective temperature in good agreement with stellar structure models. These findings are also consistent with those of an earlier broad survey of C II emission in solar-type stars (Simon and Landsman, 1991).

If the disappearance of a convective envelope implies the disappearance of a Sun-like chromosphere for hot stars, we might expect a similar change in behavior when the dynamo-generating interface between the convective zone and radiative interior disappears for fully convective, low mass stars. Initial investigations in this area focused on the so-called dMe stars, i.e., M dwarfs exhibiting H $\alpha$ emission. An early, exhaustive survey by Joy and Abt (1974) suggested that dMe stars were ubiquitous beyond the point where full convection sets in (at about spectral type M5.5), but Giampapa and Liebert (1986), using deep echelle observations of 24 late M dwarfs, observed comparable numbers of dMe and non-dMe stars, and that the $\mathrm{H} \alpha$ emission in the dMe stars was correlated with kinematic class and, by inference, with age. The existence of an activity-age relationship for these stars implied that a rotation dependent dynamo was operating even in the fully convective limit. Fleming and Giampapa (1989) later followed up these observations with a Ca K survey of $\mathrm{M}$ stars, a much harder observational task due to the very low flux; the observations also suggested the presence of a chromosphere, albeit with increasingly inefficient non-radiative heating for redder spectral types. Recent semi-empirical models of M star atmospheres (Mauas et al., 1997) indicate the presence of a chromosphere even for "basal" (i.e., the lowest activity) M stars. Chandra observations have revealed quiescent coronal emission in the M8 dwarf VB 10 (Fleming et al., 2003) (and, very recently, in an L dwarf Audard et al., 2007). The similarity of the emission in VB 10 to that of the Sun's quiet corona raises the interesting possibility that similar, non-tachocline dynamos operate in both the Sun and in extremely low-mass stars (and perhaps even brown dwarfs) where the principal cycle-generating dynamo cannot exist; it seems clear that significant magnetic flux is a pervasive component of $\mathrm{M}$ star atmospheres (Reiners and Basri, 2007).

The emphasis of this review is on "Sun-like" stars, but I also should make brief mention of the behavior of post main-sequence stars. Chromospheres are ubiquitous where subsurface convection zones are present, but Linsky and Haisch (1979), using some of the very earliest IUE data, discovered that emission we associate with transition regions and coronae was observed in giants of spectral type K1 and earlier, but not in later giants, possibly as a result of cool stellar winds. This division of giants into what Linsky and Haisch (1979) termed solar and non-solar giants initially seemed quite sharp, but more extensive samples revealed the existence of the socalled "hybrid" stars exhibiting evidence of both coronae and strong winds (Reimers, 1982; Judge et al., 1987). The division between coronal and non-coronal stars appears to apply only to giants, with all $\mathrm{G}$ and $\mathrm{K}$ giants with $\mathrm{M}_{b o l}<-2$ appearing to be X-ray sources (Reimers et al., 1996).

Rosner et al. (1995), in an exceptionally lucid Astrophysical Journal Letter, proposed that this overall behavior results from a change in the nature of the dynamo as a star evolves. Giants in the 
Linsky and Haisch (1979) solar-like category retain the large-scale dynamo we see in the modern Sun, leading to a generally Sun-like atmosphere and activity. (Along these lines, we have at least one textbook example from the long-term surveys, HD 81809, which comprises two G subgiants but exhibits a strong, well-defined 8.2 year activity cycle Baliunas et al., 1995; Hall et al., 2007b). As stars "cross" the dividing line, the activity becomes dominated by small scale fields with an open large-scale topology, permitting the development of massive winds but losing the large, closed magnetic structures associated with transition regions and coronae. Hybrid stars, which encompass a large range of coronal activity, appear to be in transition between the two stages.

Interestingly, one of the canonical "quiet" red giants, Arcturus, has been detected in X-rays by Chandra (Ayres et al., 2003), albeit with $L_{x} / L_{b o l}$ some $10^{-4}$ that of the Sun; the authors posit that the magnetic structures responsible for the emission may by the ancient giant analog of solar spicules, perhaps even responsible for driving the stellar wind itself. The details of giant star coronae are still poorly understood, but the recent observations make it clear that the magnetic nature that drives chromospheric activity on the Sun is retained by stars well past the end of their main-sequence lives.

\subsection{Going further}

Section 2.1: Students looking for a glimpse into the nature of scientific progress and debate can hardly do better than reading, cover to cover, the conference proceedings entitled Stellar Chromospheres (Jordan and Avrett, 1973). While the material is dated relative to our modern view of the chromosphere, the volume is exceptional both for its speakers' outstanding presentations of the underlying physics, as well as the unusually lengthy and detailed discussion transcripts, which present singular insights from the leading workers of the day, as well as textbook examples of spirited but professional and humor-tinged debates about the fundamental issues. This may be contrasted with the acerbic salvos between Lockyer and Huggins, who launched chromospheres research by arguing bitterly about who was doing first and best at observing the Red Flames; compare it also with the general tenor of commentary, especially among the vox populi, when solar variability is examined for its influences on terrestrial climate.

A detailed examination of how the chromosphere, transition region, and corona may be defined (along with several other topics) can be found in the thorough review by Linsky (1980). Another useful review is given by Ulmschneider (1979); see also Ulmschneider et al. (1977) and subsequent papers in the series for treatment of the generation and propagation of acoustic waves in the solar atmosphere.

Section 2.3: Excellent reviews of the consensus that emerged following the watershed of IUE and Einstein observations in the late 1970s and early 1980s are given by Linsky (1985) and Simon (1986).

Living Reviews in Solar Physics

http://www. livingreviews . org/lrsp-2008-2 


\section{The Care and Feeding of a Chromosphere}

In Section 2, I established working definitions of chromospheres and activity. I now review the development of the theory and observations that underlie the modern interpretation of chromospheric structure and activity, beginning with work of the 1960s and proceeding to the present state of affairs. The arrangement of the sections is pseudo-chronological, but the sections overlap and are otherwise not restricted in scope. The intent is to provide a general narrative describing how our picture of the energy balance and physical structure of active chromospheres has evolved since about 1960. The observational results of the long-term solar and stellar observing programs are presented in Section 4, and are linked from the sections below as appropriate.

\subsection{Early theory and models of the chromosphere}

The study of stellar chromospheric activity has evolved steadily, but has hinged on several "cusps" that have led to fundamental advances. The first of these is discussed in this section: a set of critical theoretical advances that provided a solid basis for the synoptic ground-based programs that soon followed.

In the low photosphere, plasma conditions can be determined under the simplifying assumption of local thermodynamic equilibrium (LTE), wherein the source function $\mathcal{S}_{\nu} \equiv j_{\nu} / \alpha_{\nu}$ (the ratio of emission to absorption within a given volume of plasma, or, equivalently, the emissivity per unit optical depth) saturates to the Planck function at the local electron temperature: $\mathcal{S}_{\nu}=B_{\nu}(T)$. As one moves up through the photosphere, the plasma becomes optically thin and LTE no longer applies; long range multiple scattering effects cause the source function for a line to be affected by remote as well as local conditions, diluting to some degree the responsiveness of the line profile to sharp changes in atmospheric structure, such as the initial chromospheric temperature rise. The latter appears relatively feebly in the cores of the Ca II H \& $\mathrm{K}$ lines, but much more strongly in the analogous $\mathrm{h} \& \mathrm{k}$ cores of $\mathrm{Mg}$ II (at $2800 \AA$ ); thanks to the 15 times higher $\mathrm{Mg}$ abundance, the $\mathrm{h} \& \mathrm{k}$ lines are much more opaque than $\mathrm{H} \& \mathrm{~K}$, and much less susceptible to departures from LTE at the base of the chromosphere, and thus display the temperature rise more crisply. Prior to the advent of space-based astronomy, insights about the nature of stellar chromospheric activity were therefore driven strongly by parallel theoretical and observational examinations of optical and near-UV resonance lines. The available set of lines was understandably limited, but fortunately the most prominent and best-studied of these - the $\mathrm{H}$ and $\mathrm{K}$ lines of singly ionized calcium (3933, $3968 \AA)$, the D lines of neutral sodium $(5889,5895 \AA)$, and $\mathrm{H} \alpha(6563 \AA)$ - provided precisely the observational grist needed for essential theoretical advances.

Spectral lines arise via both radiative and collisional processes, and Thomas (1957) demonstrated that in the conditions typical of a Sun-like chromosphere, the source functions of ionized metals should in general be dominated by the collisional terms, while those of neutral metals should be dominated by the radiative terms. Collisionally dominated lines such as Ca II H \& K reflect the local plasma conditions since collisional processes are obviously tied to the local electron temperature. This is manifested in the familiar emission reversal at the line cores, which for the opaque $\mathrm{H}$ and $\mathrm{K}$ lines are formed high in the chromosphere (see Figure 3); the H and K lines, therefore, have long been the obvious choice for long-term, ground-based surveys of stellar activity. Neutral metals in general reflect the continuum radiation via the control of their source functions by radiative terms, and show no such reversal. The strong $\mathrm{H} \alpha$ line, whose core is formed high in the chromosphere, is marginally photoelectrically controlled in Sun-like stars (Jefferies and Thomas, 1959; Fosbury, 1974); in the hot, high density conditions that occur during flares (or in extremely active stars) the collisional terms become increasingly important and it begins to fill in or goes into emission. The essential point to glean from all this is that the radiative versus collisional distinction is a useful first-order tool for understanding how and in which lines we might track 
stellar chromospheric activity, but the formation and nature of the lines of interest is complex and depends on the conditions over a possibly large range of depths in the atmosphere.

The extensive work on the line source function in the presence of a chromosphere, along with the "advantage of large electronic machines" as Schwarzschild (1958) quaintly but presciently put it, led to the development of non-LTE (NLTE) chromospheric models (e.g., Auer and Mihalas, 1969 and subsequent papers in that series, Athay, 1970, Vernazza et al., 1973 and subsequent papers culminating in the "VAL3" models of Vernazza et al., 1981); the models variously approached the problem of chromospheric activity analytically (i.e., calculating an emergent spectrum from first principles) and empirically (by iteratively adjusting parameters of a model to match its output to an observed temperature distribution). Among other things, these models made it apparent that significant mechanical heating was necessary to sustain observed chromospheric conditions (Athay, 1970). An important (but understandable) shortcoming of these models was their temporal and spatially-averaged nature; these shortcomings and further details about the development of chromospheric modeling are discussed in Section 3.4.

\subsection{Long-term observations of Ca II $\mathrm{H} \& \mathrm{~K}$}

The strongest spectral features observable from the ground are those dubbed H and K by Fraunhofer in 1814, arising from singly ionized calcium. Eberhard and Schwarzschild (1913) noted the presence of pronounced emission cores in these lines in spectra of Arcturus and other stars, and sensibly wondered (1) whether the emission arose from processes analogous to solar activity and (2) if the emission varied periodically in a manner analogous to the sunspot cycle. Perhaps no researcher pursued these questions with such patience and perseverance as Olin Wilson at the Mount Wilson Observatory (MWO; the confluence of names is a coincidence). Wilson's initial synoptic observations revealed that the chromospheric activity of main-sequence stars decreases with age (Wilson, 1963; Wilson and Skumanich, 1964); this result was eventually synthesized in the famous paper by Skumanich (1972), in which the stars' Ca II emission (and by inference, the mean surface magnetic field) was shown to decay as the inverse square root of the age.

The early MWO observations also revealed a curiously linear relation between the absolute magnitude and the $\log$ of the $\mathrm{K}$ line emission widths for $\mathrm{G}$ and later stars, dwarfs, and giants alike: $M_{v}=27.59-14.94 \log W_{o}(\mathrm{~K})$ (Wilson and Bappu, 1957). Relationships similar to the "Wilson-Bappu effect" were subsequently found for other resonance lines, such as Mg II k $\lambda 2796$ and Ly $\alpha \lambda 1216$ (McClintock et al., 1975; Weiler and Oegerle, 1979). Numerous explanations were advanced, but Ayres (1979), using scaling laws for the extent and density of the chromosphere, argued that the Wilson-Bappu width is "a stellar barometer, not a tachometer"; i.e., rather than arising from details of chromospheric flows, it is primarily a hydrostatic equilibrium acting in concert with the partial ionization of hydrogen and resonance line cooling to postpone the onset of the sharp thermal instability that gives rise to the corona. Pace et al. (2003) suggested that the Wilson-Bappu relationship can be used to infer cluster distances, though it contains too much scatter to be useful for individual stellar distances.

In 1966, Wilson undertook a systematic program of Ca II H \& K observations of main-sequence stars, and in a seminal paper (Wilson, 1978) posed the question Does the chromospheric activity of main-sequence stars vary with time, and if so, how? In that paper he concluded that all stellar chromospheres were variable to one extent or another, that cyclical variations very likely did exist, and that they should be considered to be generated and dissipated by processes and structures analogous to those observed on the Sun until proven otherwise. Following Wilson's retirement, the so-called "HK Project" continued under the direction of S. Baliunas until the end of 2003, and it remains today the fundamental observational data set of stellar chromospheric activity. Further details about the program and its major results, as well as other stellar cycles investigations, are presented in Section 4.1.

Living Reviews in Solar Physics

http://www. livingreviews . org/lrsp-2008-2 
Complementing the stellar cycles programs are long-term HK observations of the Sun, both resolved and full disk (i.e., "the Sun-as-a-star"). Wilson observed the Moon as a solar proxy with the MWO spectrometer, and early observations of the solar Ca K line suggested that the central $\mathrm{K}_{2}$ emission peak varied by as much as $40 \%$ over the solar cycle (Sheeley Jr, 1967). Direct, longterm H \& K observations of the Sun began at the National Solar Observatory (NSO) in 1974 using the McMath solar telescope at Kitt Peak (White and Livingston, 1978), and in 1976 at Sacramento Peak (Keil and Worden, 1984). By the peak of solar cycle 21, it was apparent that the "HK index," the total emission in a $1 \AA$ rectangle centered on the line cores, closely tracked the sunspot number, plage index, and $10.7 \mathrm{~cm}$ flux (White and Livingston, 1981). The NSO workers also monitored a number of photospheric features (e.g., C I $\lambda 5380$ and several iron lines) to study the relationship between chromospheric activity, photospheric structure, and solar and stellar luminosity (Livingston et al., 1977; Livingston and Holweger, 1982; White et al., 1987); see also Section 4.6.

\subsection{From ground to space}

The second cusp in our understanding of stellar chromospheric activity occurred beginning in 1978, with the deployment in rather rapid sequence of a number of space-based observatories, especially the International Ultraviolet Explorer (IUE) and the Einstein X-ray observatory; although certainly not the first observational ventures into space, they were far more capable than their predecessors. The advent of these satellites had two effects: (1) continuous access to the UV and X-ray spectrum where most outer atmosphere diagnostics lie, and (2) a steadily growing data set of flux-calibrated spectra. Thus, although the nature of IUE and similar missions precluded the kind of longterm observations Olin Wilson was able to carry out, a watershed of observations of outer stellar atmospheres rapidly accumulated.

A critical early result was the discovery of tight power law correlations between emission in chromospheric, transition region, and coronal lines (e.g., Ayres et al., 1981). A few quotations from that paper loom large in the present-day literature; Ayres et al. (1981) suggest that "the very existence of the correlations argues that coronae and chromospheres are physically associated," and later in their paper, discussing chromospheric and coronal heating mechanisms, they write that "the small-scale magnetic flux tubes thought to comprise the major component of the solar surface field [may] serve as conduits of wave energy (acoustic or Alfvén) into the outer atmosphere... While magnetic fields very likely provide the structure of stellar chromospheres, they may be responsible only indirectly for the heating". This is a fundamental shift in thinking from the time averaged, spatially averaged nature of the "first generation" models discussed above.

Subsequent work demonstrated that similar power laws applied for numerous diagnostics for normal dwarfs to binaries and even bizarre objects such as FK Comae stars (Oranje, 1986). Wilson's Ca II H \& K measurements also were shown to be similarly related to transition region and coronal emission (Oranje and Zwaan, 1985; Schrijver, 1987), solidifying the idea of a tight physical relationship between the "layers" of the outer stellar atmosphere. For an exhaustive treatment of progress in the early IUE years, see the review by Jordan and Linsky (1987).

Progress during this period was not limited to the stars. Within months of the commissioning of IUE, the Nimbus-7 satellite was launched, initiating what is now a three-decade record of spacebased measurements of the total solar irradiance (TSI). The extended TSI composite appears in Figure 4; this figure is widely reproduced but of sufficient importance to the interpretation of stellar activity and luminosity that I include it here. Further arguments are deferred to Section 4.7; suffice to say here that the discovery that the solar constant isn't, as Figure 4 clearly shows, created an important new dimension to the study of stellar activity, and is a pivotal part of the radical transformation of the field circa 1980. 


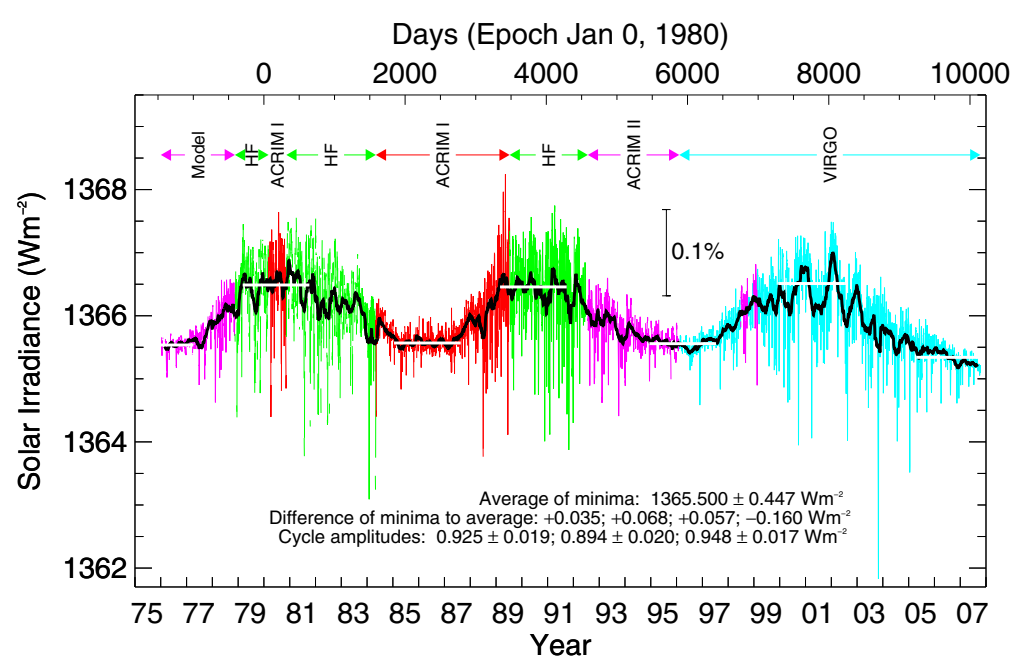

Figure 4: The extended composite total solar irradiance (TSI) record since 1975, from the World Radiation Center (PMOD-WRC) in Davos, Switzerland (Fröhlich).

\subsection{Semi-empirical models}

Empirical chromospheric models, in which parameters are adjusted until a satisfactory fit with observations is obtained, began to emerge in earnest in the 1970s as logical outgrowths of extensive work on the solar CaII line. Initial efforts in this area began with the first of the papers in the important Stellar Model Chromospheres (SMC) series by Jeffrey Linsky and his 'Cool Star Mafia' (his term, not mine). Their early models focused on Procyon (Ayres et al., 1974) and Arcturus (Ayres and Linsky, 1975), since these were at that time the only stars other than the Sun with high resolution, absolutely calibrated atlases suitable for creating absolute flux profiles of the Ca II lines. In subsequent papers, these models were refined and extended to dwarfs spanning the right half of the HR diagram (Ayres et al., 1976; Kelch, 1978; Giampapa et al., 1981).

These early models provided a number of fundamental insights on the broad characteristics of stellar atmospheric structure; perhaps most significantly, evidence was found for non-radiative heating in the photosphere as well as the chromosphere, and both the temperature gradient and the radiative loss rates were found to be higher for active chromosphere stars than for "quiet" stars. These modeling efforts also necessitated the development of methods for creating absolutely (if approximately) flux-calibrated profiles of a large number of cool stars (see Section 4.2). However, they also had some critical limitations, and Ayres and Linsky (1975) acknowledge these explicitly in their work on Arcturus: "A more detailed model [of the upper photosphere and low chromosphere] could be devised, but we feel that such an approach is not justifiable until we have a reliable estimate of the extent to which our assumptions of a homogeneous, static, plane-parallel chromosphere are valid for Arcturus ...".

This turned out to be a wise caveat. The "simplicity" of the early models was driven, of course, by the limitations of the available observations, both in terms of resolution and wavelength. As new data gradually overcame these limitations, it became clear that one-component models could not explain the full complexity of the chromosphere, a classic example being Heasley et al. (1978), who demonstrated that the derived parameters in the Ayres \& Linsky model could not replicate Arcturus's $\mathrm{CO}$ and $\mathrm{Ca} \mathrm{K}$ line wing spectrum. This provided compelling evidence for the inhomogeneous nature of the chromosphere summarized in Section 2.1, and served as a natural springboard for more advanced modeling (see, e.g., Uitenbroek, 2000).

As the IUE era got underway, the growing body of ultraviolet data permitted much more

Living Reviews in Solar Physics

http: //www. livingreviews.org/lrsp-2008-2 
extensive investigation of stellar chromospheres and transition regions, spurring two additional important series of papers: Outer Atmospheres of Cool Stars (Linsky and Haisch, 1979 and subsequent papers), continuing the semi-empirical modeling efforts in the SMC series, and Magnetic Structure in Cool Stars (Middelkoop and Zwaan, 1981 and subsequent papers). The former series focuses extensively on individual stars modeled in the SMC era, while the latter provides arguably the most comprehensive examination in the literature of the Mt. Wilson $\mathrm{H} \& \mathrm{~K}$ data with the space-based data, but a pervasive theme in both series is the continual discovery of structures in stars that appeared to be direct analogs of solar outer atmospheric structures.

Semi-empirical models remain the standard approach to understanding chromospheric structure today, with the older "VAL" models having been supplanted by the "FAL" models (e.g., Fontenla et al., 1993). First-principle, theoretical models of the chromosphere, incorporating the effects of magnetic fields, appear to be the "next wave," though their full 3D incarnations remain beyond current computational limits (see the review by Carlsson, 2007 for a detailed discussion).

\subsection{The high resolution chromosphere}

In the preceding sections, I have reviewed the development of ground-based observations and models for phenomena that are clear signatures of chromospheric heating via non-thermal mechanisms. In this section I review the physical arrangement of these phenomena in the Sun, as revealed by modern, high-resolution instrumentation, under the assumption that analogous structures are responsible for stellar chromospheric activity, and that a detailed view of solar features will provide a more visceral feel for the unresolved observations of their stellar analogs.

Stellar activity cycles have long been considered to be the result of a self-sustaining magnetic dynamo, created by the combined action of turbulent convection and differential rotation (e.g., Parker, 1955; Babcock, 1961), and are likely generated near the base of the convection zone (in the Sun, $\approx 1.5 \times 10^{5} \mathrm{~km}$ below the visible surface; Parker, 1975). Chromospheric activity can be a useful proxy for luminosity variations, since the structures responsible for both are dependent on the extent and distribution of this magnetic field. The total solar irradiance varies by $\approx 0.1 \%$ over the activity cycle (Fröhlich and Lean, 1998), primarily as a result of darkening by sunspots but brightening by emission from faculae and network. At left in Figure 5, the familiar sunspots (regions of intensely concentrated magnetic flux emerging from the solar interior) and faculae (in visible light best seen near the limb, where their warmer high altitude layers are more evident due to the oblique viewing angle, see Keller et al., 2004) are apparent. Spruit (1977) gives a classic treatise on these phenomena, describing spots as "shadows" on the solar surface arising from the suppression of convective heat transport by the intense, concentrated magnetic fields. These elements permeate the chromosphere, and are apparent in the CaII $\mathrm{K}$ image at right in Figure 5; numerous bright chromospheric emission regions, or plage, are present.

The "quiet Sun" away from active regions is also permeated by magnetic features. High resolution images of the quiet photosphere appear in Figure 6, obtained by the Dutch Open Telescope (DOT, left) and the Swedish Solar Telescope (SST, right). The photospheric granulation is obvious in the patterns of bright granules and dark intergranular lanes, as is the filamentary structure of the sunspot penumbra. The SST observations near the limb at oblique viewing angles (e.g., Lites et al., 2004) clearly reveal the granules, which are buoyant convective bubbles arriving at the solar surface, as three-dimensional "hills;" they release their energy once the gas is sufficiently optically thin, and the cooled plasma then sinks back into the interior in the dark lanes. Myriad tiny magnetic flux tubes emerge in the granule centers, are swept up by horizontal flows, and collect in the intergranular lanes (e.g., Spruit and Roberts, 1983), often appearing as bright points in $\mathrm{CH}$ ("G band") images: their lower internal gas densities suppresses the molecular formation and allows radiation from hotter deeper layers to escape (see Figure 7, left).

The images in Figure 8 provide an exquisite view of the solar atmosphere from photosphere to 


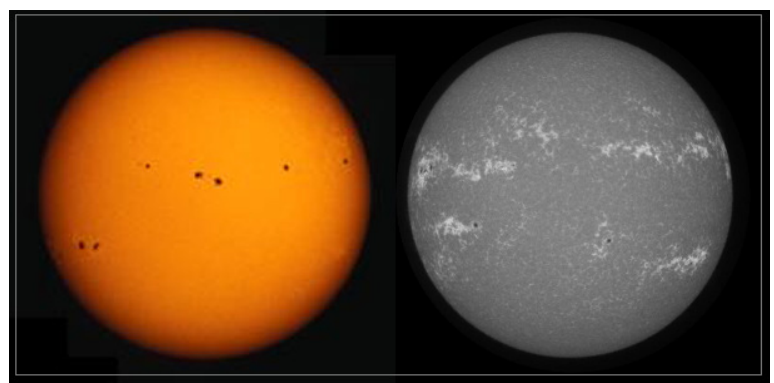

Figure 5: Left: a well-known white light image of the Sun, illustrating sunspots and faculae, the latter being apparent as bright regions near the limb (image courtesy NSO/AURA/NSF). Right: Ca II K image showing chromospheric emission from active regions and network (image courtesy Big Bear Solar Observatory/New Jersey Institute of Technology). The chromospheric extensions of the faculae (the so-called "plage") are readily visible across the disk.

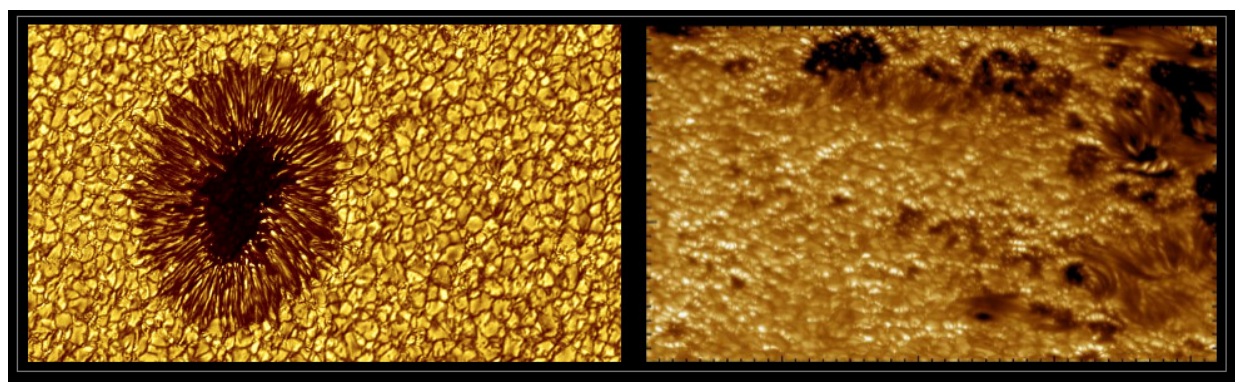

Figure 6: Left: A closeup of a sunspot, clearly showing the penumbral spot structure and surrounding photospheric granulation (image courtesy Dutch Open Telescope). Right: a sunspot, granulation, and faculae observed from a more oblique angle (image courtesy Goran Scharmer and Mats G. Löfdahl, Institute for Solar Physics of the Royal Swedish Academy of Sciences).

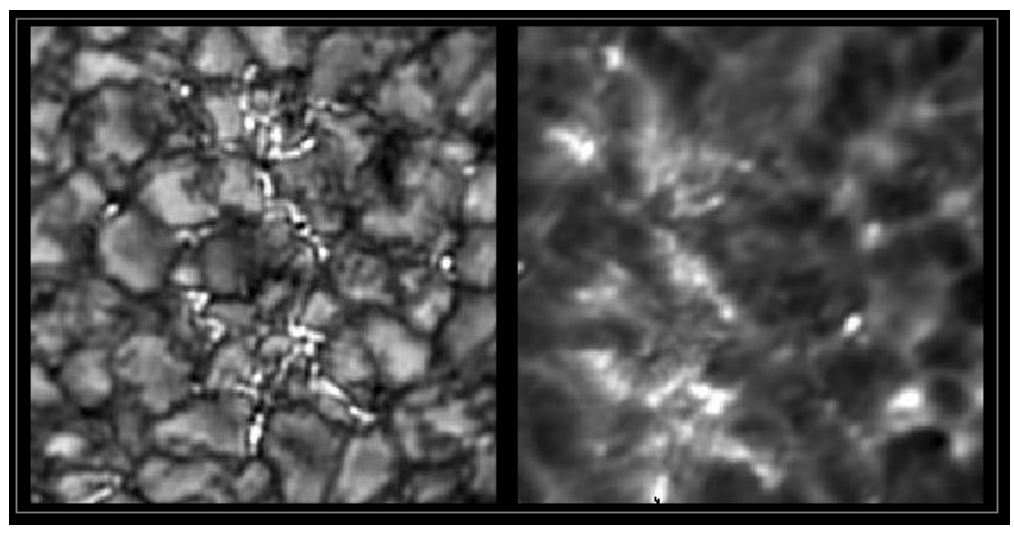

Figure 7: Left: Observations of photospheric granulation in the CH G band (4300 $)$ show the granulation and bright points in the dark lanes; these are where the line of sight intersects flux tubes whose rarefied interiors allow one to see to deeper, hotter gas. Right: the upper photosphere, viewed in the Ca II H line, exhibits reversed granulation (images courtesy Dutch Open Telescope). 
chromosphere, obtained with the Dutch Open Telescope and the Transition Region and Coronal Explorer (TRACE). At top left is the photosphere, showing the very large active region AR10486, marked by a number of spots and associated faculae. The simultaneous image of the chromosphere at top right clearly reveals the greatly enhanced emission in the magnetically active areas near the active region, as well as the general manifestation of the chromosphere as a seething mass of narrow, magnetically confined structures. In this image, the Ca II H primarily samples verticallyoriented flux tubes that have been called straws (Rutten, 2007a) extending into hot transition region and coronal regimes; an $\mathrm{H} \alpha$ image reveals somewhat different but still discrete structures variously termed mottles, fibrils, and spicules (e.g., Suematsu et al., 1995). This is therefore a highly resolved version of what the long-term activity cycle programs discussed in Section 4.1 "see" in the various chromospheric activity indices. Finally, at the bottom in Figure 8 is an image taken by TRACE of AR10486 shortly after an enormous X-class flare that erupted two days after the images at top were taken. An arcade of cooling, post-flare loops is present; these delicate, croquet wicket structures are found not only in the active corona, but also in "quiet" regions (see Schrijver et al., 1999 for an overview of initial TRACE observations).

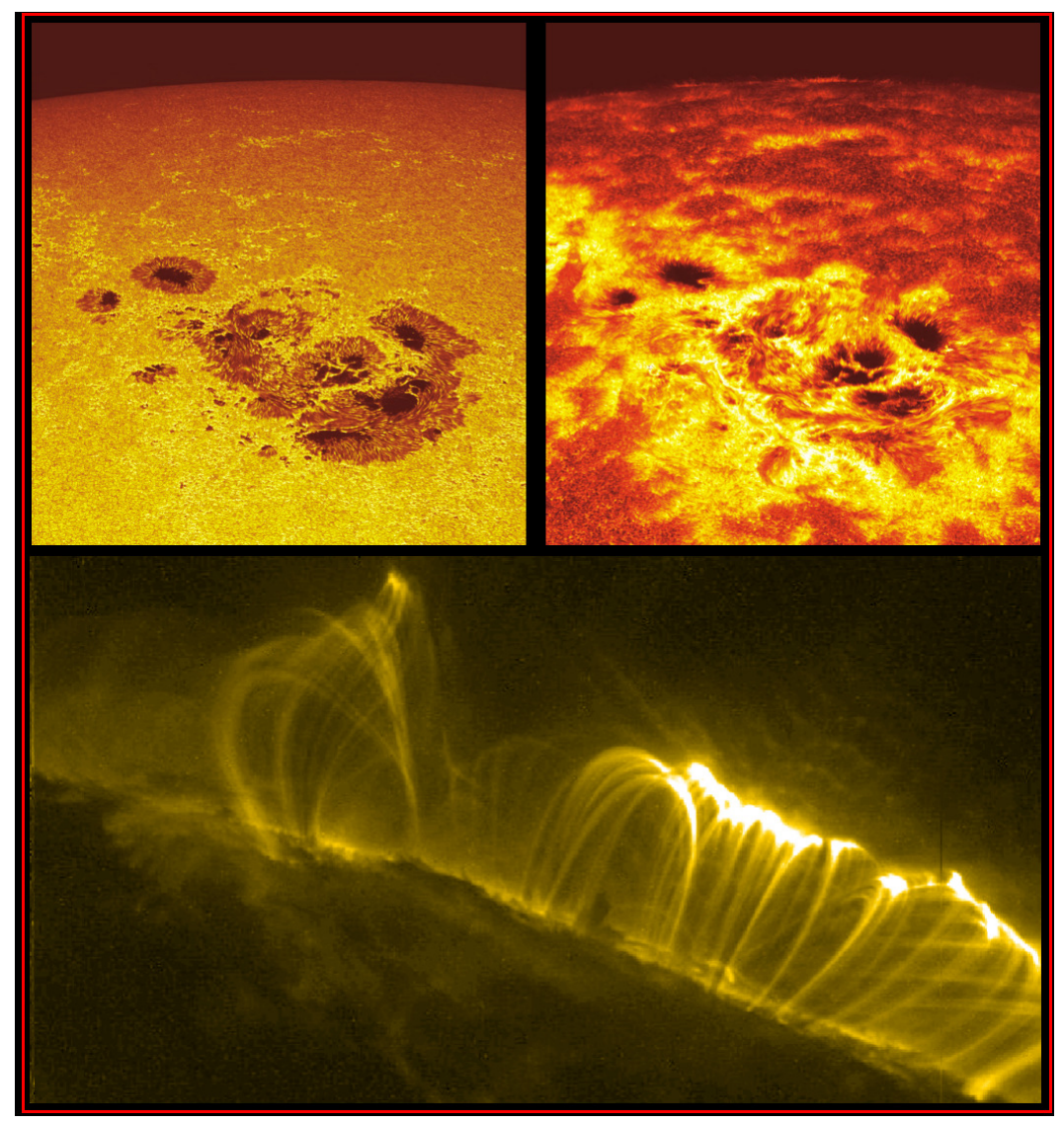

Figure 8: Three contemporaneous views of the Sun. Top left: The solar photosphere, observed in the light of the $\lambda 4300 \mathrm{CH} \mathrm{G}$ band on 2003 November 2. Active region AR10486 is apparent at center. Top right: The solar chromosphere in the same location and time, observed in Ca II H (images courtesy Dutch Open Telescope). Bottom: A $\lambda 171 \AA$ image of an arcade of magnetic loops following a large flare that erupted from AR 10486 two days after the other images were taken (image courtesy Transition Region and Coronal Explorer). 


\subsection{The modern chromosphere}

Figure 8 represents what we may call a third cusp in our view of solar and stellar chromospheres. So far, we have little evidence that stellar active chromospheric structures are substantially different from the Sun's, and the data from TRACE, SOHO, Hinode, SORCE, and comparable missions must strongly guide stellar activity research over the next 15 years.

The earliest TRACE data revealed the highly confined nature of the outer solar atmosphere (Schrijver et al., 1999), and have bolstered a "dynamical" view of the chromosphere that contrasts sharply with the "layered" view alluded to in Section 2, and as modeled by the workers of the 1960s and 1970s (Section 3.1). Carlsson and Stein (1995) even asked "Does a non-magnetic solar chromosphere exist?", arguing that in the internetwork regions, the temperature rise is merely an artifact of time-averaged modeling, with evanescent pulses of high temperatures occurring from periodic heating by wave dissipation. This view is supported by the presence of cool CO $4.7 \mu \mathrm{m}$ emission at chromospheric heights, the amusingly named CO-mosphere (Wiedemann et al., 1994). This interpretation has met with criticism (Kalkofen et al., 1999; Kalkofen, 2001), but Ayres (2002) argues that much of what was classically considered the chromosphere is indeed quite cool, and Rutten (2007b) goes so far as to suggest that the chromosphere comprises merely the $\mathrm{H} \alpha$ mottles and fibrils - which leads us full circle to the beautiful pink annulus of Figure 1, though with a far different conceptual basis.

With this somewhat revised view to a chromosphere, I review in Section 4 the principal results from long-term observations of solar and stellar chromospheric activity.

\subsection{Going further}

Section 3.1: The size of the literature makes any statement like this rather subjective, but I believe the student interested in the development of stellar atmospheres theory can profitably use the beautiful trio of papers by Thomas (1957), Jefferies and Thomas (1958), and Jefferies and Thomas (1959) as an essential node both for researching subsequent developments as well as the early work by Milne, Eddington, Chandrasekhar, and others. These papers are singularly illuminating and readable. The early models have of course been thoroughly superceded; the classic VAL (Vernazza et al., 1981) models may be compared, for example, with Anderson and Athay (1989), the "FAL" stars (Fontenla et al., 1993 and previous papers in the series), and fully 3D models of "dynamic" chromospheres that are coming within the reach of current computing power (for a recent review, see Carlsson, 2007).

Section 3.2: Olin Wilson's early explorations of the relationship between stellar rotation, chromospheric activity, and age used observations of clusters (the Hyades, Pleiades, Praesepe, and Coma, Wilson, 1963), but were soon extended to field stars (Wilson and Skumanich, 1964), many of which would end up on his long-term stellar cycles program. The concept of "chromospheric ages" as deduced from the Ca II H \& K activity proxy has since received a great deal of attention; an important synthesis is presented by Soderblom et al. (1991), and Wright et al. (2004) give a large set of chromospheric ages for stars observed as part of the Carnegie Planet Search Project. Wilson's long-term observations of stellar chromospheric activity commenced in March 1966 with 139 stars selected not for any particular "Sun-like" nature, but by virtue of noticeable HK emission in spectrograms he had previously obtained (this point will return when I discuss solar analogs later in this article). Even after over a year of observing, Wilson was evidently not sanguine about the results of the program, noting that "If Sheeley's [Sheeley Jr (1967), who found the solar K line central intensity varied by $40 \%$ over the activity cycle] results are correct ... the observational problem of finding stellar cycles should not be too difficult. However, the experiences of Popper and myself suggest a more pessimistic outlook" (Wilson, 1968).

Section 3.5: Details of dynamo research and its numerous outstanding questions are outside the scope of this review; for a recent evaluation see Charbonneau (2005), and for summaries of

Living Reviews in Solar Physics

http://www. livingreviews . org/lrsp-2008-2 
the long-term observational programs within the dynamo context, see Baliunas et al. (1996) and Strassmeier (2005).

Section 3.6: In several places in this review, I make note of the evanescent nature of the chromosphere that these recent observations clearly support. I thank one of the referees for suggesting a comparison in this regard that should be useful for both researchers and students: despite the volume over which it is distributed, the mass of the solar chromosphere is only about the mass of Earth's atmosphere; moreover, the mass of the photosphere down to $\tau=1$ in the continuum is only about that of the Indian Ocean! 


\section{Chromospheric Activity}

Section 3 presents a synopsis of the development and present state of our understanding of active chromospheric structures. In this section, I will discuss some of the major observational programs and their results.

As discussed in Section 3.2, the Mount Wilson program was initially established to answer the long-standing question of whether there were stellar analogs of the solar activity cycle. At the same time, the theoretical basis was developing for understanding activity cycles as manifestations of a magnetic dynamo driven by differential rotation and the convective envelopes of Sun-like stars. Increasingly detailed observations of the Sun's magnetic field also revealed its organization into magnetic structures of varying size, from larger active regions to smaller network components. A fundamental astrophysical motivation for observations of stellar chromospheric activity, therefore, is the broader understanding of the operation of stellar dynamos and, with the advent of very high-resolution observations and analysis techniques such as Doppler imaging, the distribution of the magnetic structures responsible for activity around the stellar surfaces. Understanding the nature and evolution of dynamo processes in the cool half of the Hertzsprung-Russell diagram, and their effect on stellar output in various wavelength regimes and over the evolutionary history of the stars, remains an essential impetus for this work.

Observations of chromospheric activity developed an additional motivation with the launch of space-based observatories and the realization that the Sun (i) did not have constant luminosity and (ii) exhibited increasingly large variations over its activity cycle toward shorter wavelengths, particularly in the X-rays. Combined with the renewed awareness and interest in Maunder Minimum episodes (Eddy, 1976), the obvious possibility that solar variations might have some impact on terrestrial climate created a keen interest in identifying genuine twins of the Sun, whose chromospheric activity would ostensibly be the most nearly valid proxy for assessing the likely envelope of solar variability. The solar analog hunt was begun by Hardorp (1978), and in the best tradition of astronomical research, it grew into a cottage industry (see, e.g., Friel et al., 1993 and previous papers in the series, the exhaustive review by Cayrel de Strobel, 1996, and the "Top Ten" solar analogs of Soubiran and Triaud, 2004). Understanding the so-called "solar-stellar connection" through high-resolution solar observations and proxy stellar observations remains an important focus of stellar activity work today, especially given the apparent paucity of real solar twins (see Section 5 for further thoughts).

\subsection{Synoptic observations and surveys of Ca II $\mathrm{H} \& \mathrm{~K}$}

Our understanding of the long-term behavior of stellar chromospheric activity comes primarily from the 40-year HK Project at Mount Wilson Observatory (MWO), which operated from 1966 through 2003; major summaries of the observations are given by Wilson (1978), Duncan et al. (1991), and Baliunas et al. (1995). Observations from the HK Project are expressed in terms of the dimensionless index $S$, the ratio of emission in the $\mathrm{H} \& \mathrm{~K}$ lines cores to that in two nearby pseudocontinuum reference bandpasses (see Section 4.2 for details). The HK data have been used to explore a variety of stellar properties; in this section, I summarize the major observational programs and the general stellar ensemble properties and periodicities present in the data sets.

Baliunas et al. (1998) found that $60 \%$ of stars in the MWO survey exhibited periodic, cyclic variations, $25 \%$ showed irregular or aperiodic variability, and $15 \%$ had flat activity records (see Figure 9). Within these broad classifications are stars that also show evidence for multiple periodicities (Baliunas et al., 1995). This general distribution of cycle characteristics is also found in the complementary long-term Solar-Stellar Spectrograph (SSS) program at Lowell Observatory, but in a target set more closely clustered on the most nearly Sun-like stars than in the MWO sample (Hall et al., 2007b). In general, in the MWO stars both the amplitude and the mean level

Living Reviews in Solar Physics

http://www. livingreviews.org/lrsp-2008-2 
of chromospheric activity decrease with increasing cycle length, and cycles shorter than about 6 yr in Sun-like stars are not observed (Baliunas and Soon, 1995).
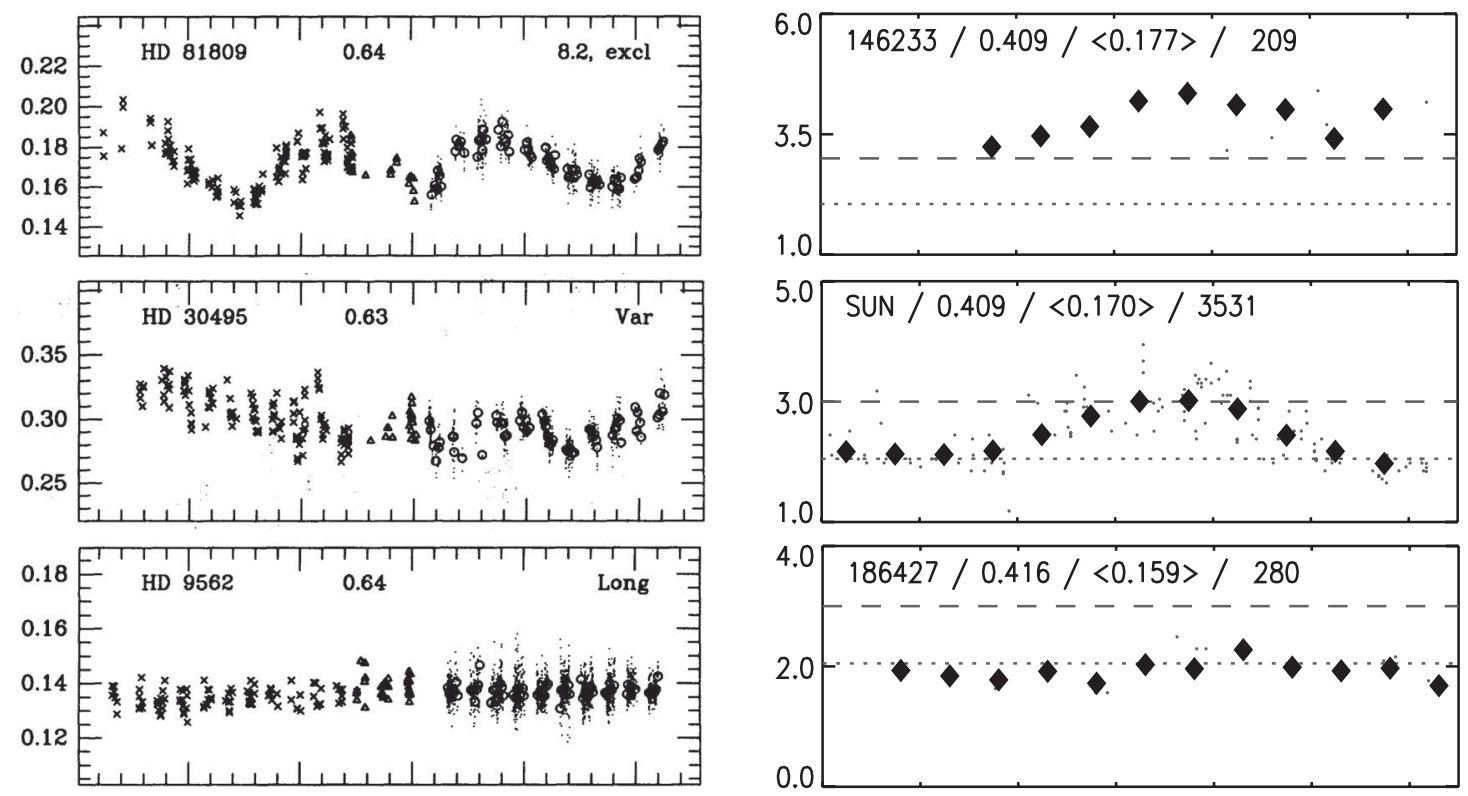

Figure 9: Left: Representative time series from the MWO HK Project, illustrating periodically variable (top), irregular (center), and flat (bottom) chromospheric activity, expressed in terms of the dimensionless $S$ index, running from 1966-1991 (from Baliunas et al., 1995). Right: Analogous series from the Lowell Observatory SSS project, showing the flux-calibrated time series for the Sun (top), the cycling solar twin 18 Scorpii = HD 146233 (center), and the relatively inactive solar analog 16 Cygni B = HD 186427. Quantities in brackets at the top of each panel are the $S$ values derived from the spectra. The time span of the SSS series is 1994-2006 (from Hall et al., 2007b).

In addition to identifying cycle durations, the MWO series can be used to identify rotation periods and even differential rotation via drifts in rotation-timescale periodicities in $S$, assuming that the emergence sites of stellar active regions migrate in latitude as do their solar counterparts, and if their evolution does not obscure the signal. An initial attempt to extract the rather weak differential rotation signal from the MWO series had limited success (Baliunas et al., 1985), but Donahue and Baliunas (1992) reported detection of a drift in the apparent rotation period in $\beta$ Com $=$ HD 114710. The sense of the change, however, was opposite that of the Sun, with longer periods late in the cycle (from the familiar "butterfly" progression of active region latitudes over the solar cycle, we find shorter periods as late-cycle active regions migrate toward the solar equator). Obviously, the more active the star, the more likely such detections will be. Photometric Doppler imaging techniques have been usefully applied to a number of very active stars (e.g., Strassmeier, 1996 and subsequent papers in the series), but Hempelmann (2003) argues that the HK proxy and similar diagnostics such as Mg II and Ly $\alpha$ are more fruitful avenues for this work in low-activity, Sun-like stars, since they are not affected by the cancellation of active region brightening and spot darkening.

The MWO and Lowell data sets are magnitude-limited in their high-priority target list to about $m_{V}<7$ due both to instrumental limitations and the necessity of maintaining a relatively high observing cadence on samples of $\approx 100$ stars, and they have been supplemented by a number of complementary programs that both reach somewhat fainter, as well as providing broad snapshots of patterns in chromospheric activity. 
Wright et al. (2004) used the Carnegie Planet Search data set to derive $S, R_{\mathrm{HK}}^{\prime}$, chromospheric ages, and rotation rates for the largest survey sample presented to date, with results for over 1200 F-M stars.

A library of flux-calibrated spectra of 91 southern Sun-like stars has been published by Cincunegui and Mauas (2004); as I will discuss in Section 4.2, such libraries are certainly a desirable trend and should be expanded.

Giampapa et al. (2006) surveyed the CaII H \& K emission of a sample of F-K stars in the open cluster M67, probing a roughly solar-age group of dwarfs at much fainter magnitudes than the MWO or Lowell programs. The chromospheric activity of the M67 stars was comparable to that observed in modern solar cycles, though $\approx 25 \%$ of the stars were found outside the modern solar cycle excursion. About $15 \%$ of these were found to have activity levels below the present solar minimum, which is comparable to the frequency of flat activity stars in the MWO and Lowell surveys. It is not clear, however, if these are truly in non-cycling states, or merely represent the minima of cycling stars.

Henry et al. (1996) surveyed the Ca II H \& K activity of over 800 southern Sun-like stars, a sample mostly independent of the synoptic northern hemisphere programs. The general characteristics of the ensemble appear in Figure 10, and they are typical of the activity-color appearance of samples of Sun-like stars. Henry et al. (1996) identified four qualitative activity classes ranging from "very active" to "very inactive," as shown in the left panel of Figure 10. They also found that (1) there was a deficiency in stars at intermediate levels of activity and that (2) this led to a bimodal distribution of activity (right panel of Figure 10) that could be explained by a double Gaussian distribution of active and inactive Sun-like stars, but which also revealed a very lowactivity excess $\left(\log R_{\mathrm{HK}}^{\prime}<-5.1\right)$ which they suggested might be stars in Maunder Minimum-like states.
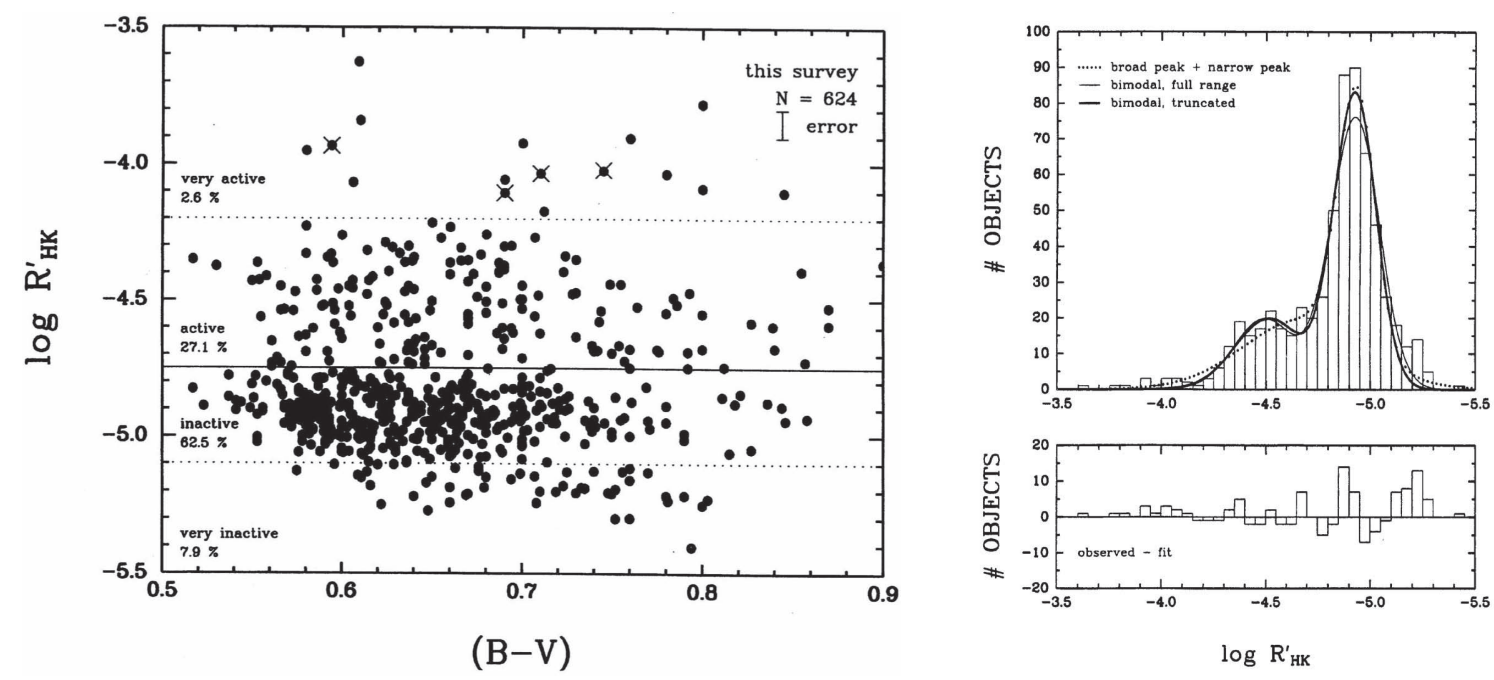

Figure 10: The distribution of activity in 815 southern Sun-like stars. Left panel: color versus activity, displaying four qualitative activity levels and a relative lack of intermediate-activity stars of $R_{\mathrm{HK}}^{\prime} \approx-4.65$. Right panel: the distribution of activity is bimodal, though with a small low-activity excess that may represent Maunder Minimum stars (from Henry et al., 1996).

A more recent survey undertaken as part of the NStars project is discussed by Gray et al. (2003) and Gray et al. (2006). In their analysis of their northern sample, Gray et al. (2003) obtained a bimodal activity distribution essentially identical to that of Henry et al. (1996), including a significant excess of possibly Maunder Minimum stars at $R_{\mathrm{HK}}^{\prime}=-5.1$. Further work (Gray

Living Reviews in Solar Physics

http://www. livingreviews.org/lrsp-2008-2 
et al., 2006) revealed that the apparently ubiquitous bimodal distribution was in fact a function of metallicity (see Figure 11). Metal-poor stars of $[\mathrm{M} / \mathrm{H}]<-0.2$ do not exhibit a bimodal activity distribution. Although the interpretation is complicated by the presence of a number of active stars in the metal-poor sample, Gray et al. (2006) speculate that the pronounced break between bimodal and unimodal activity distributions at $[\mathrm{M} / \mathrm{H}]=-0.2$ may reflect a physical change that allows the presence of active chromospheres for solar-metallicity stars, while suppressing them in metal-deficient stars. Further aspects of the "break" between active and inactive stars are noted in Section 4.3.
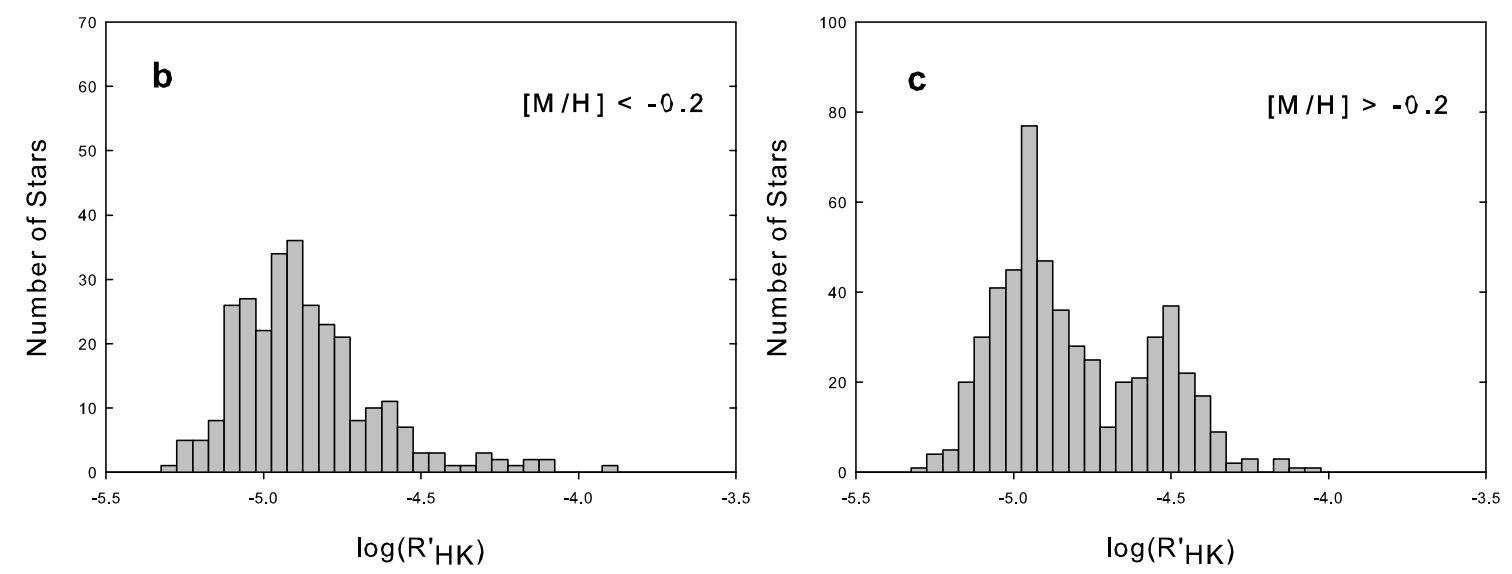

Figure 11: The distribution of activity F, G, and $\mathrm{K}$ dwarfs as a function of metallicity. Metal-poor stars show a singly-peaked distribution (left panel), while for stars with $[\mathrm{M} / \mathrm{H}]>-0.2$, the distribution is bimodal (from Gray et al., 2006).

\subsection{Measuring chromospheric activity}

The glut of space-based observations of chromospheric activity from IUE were calibrated in absolute flux units, which had important consequences. Estimates of energy balance in the chromosphere naturally emerged in physical units from the models, but determining the analogous quantities from the ground-based observations is notoriously more difficult. Absolute flux calibrations rely on the primary spectrophotometric standards Vega and 109 Vir (Hayes and Latham, 1975; Tüg et al., 1977) or a handful of secondary standards (Hayes, 1970), and are especially vexing to relate to heavily blanketed continua such as the blue and near UV regions of Sun-like stars. Additionally, the usual measures of $\mathrm{Ca} \mathrm{H} \& \mathrm{~K}$ and $\mathrm{H} \alpha$ contain significant photospheric flux leaking into the filter bandpasses, and determining exactly what to remove is non-trivial. This difficulty heavily influences the characterization of ground-based observations of chromospheric activity the reader will find in the literature.

Absolute Ca K chromospheric fluxes for cool main-sequence stars were computed by Blanco et al. (1974) and Blanco et al. (1976), but Linsky and Ayres (1978) showed that their method underestimated the chromospheric radiative losses in the $\mathrm{K}$ line. Linsky et al. (1979) created absolute $\mathrm{Ca} \mathrm{H} \& \mathrm{~K}$ flux profiles of F-M dwarfs and giants by deriving empirical relations giving the $\lambda 3950$ surface flux as a function of Johnson $V-R$. This work was the most extensive absolute flux reference against which the initial space-based observations were compared, and other workers have adapted the same procedure to produce absolutely calibrated data sets for both H \& K (e.g., Pasquini et al., 1988) and $\mathrm{H} \alpha$ (Pasquini and Pallavicini, 1991).

In Sections 3.2 and 4.1, I discussed the origin and principal results of the four-decade Mount 
Wilson Observatory (MWO) survey of chromospheric activity in late-type stars. The HK photometer used in the initial MWO survey (Wilson, 1978) was replaced in 1977 by a successor instrument, the "HKP-2," but both instruments measured activity in the same way and were satisfactorily cross-calibrated in terms of the nearly ubiquitous activity index $S$ (Vaughan et al., 1978). This is a dimensionless ratio of the emission in the line cores to that in two nearby continuum bandpasses on either side of the $\mathrm{H}$ and K lines: $S \propto\left(\mathcal{F}_{H}+\mathcal{F}_{K}\right) /\left(\mathcal{F}_{R}+\mathcal{F}_{V}\right)$. The $S$ index obviates the need for absolute flux calibration while still providing a self-consistent assessment of activity for the Sun or a given star, but it has a color term (from the reference bandpasses) and also includes a photospheric contribution in the line core bandpasses; thus, it is difficult to interpret trends in $S$ for a stellar ensemble, and challenging to tie it meaningfully to model predictions.

This has led a number of authors to explore the conversion of $S$ to physical units. Middelkoop (1982) developed a method to remove the color term from $S$, and Noyes et al. (1984) extended this work to create the dimensionless index $R_{\mathrm{HK}}^{\prime}$, which essentially gives the fraction of a star's bolometric luminosity radiated as chromospheric H and K emission. Nearly as common in the literature as $S, R^{\prime}$ is typically expressed in log units and ranges from about -4.4 to -5.1 for very active to highly inactive stars. To some extent, however, even $R^{\prime}$ is unsatisfactory due to luminosity and metallicity effects, and Rutten and Schrijver (1987) found that the surface flux density was the optimum unit to use for deriving relations between activity proxies (e.g., soft X-rays and Mg II $\mathrm{h}$ $\& \mathrm{k}$ ), as well as relationships between activity and rotation.

The calibration of $S$ to flux has been primarily achieved using empirical approaches (e.g., Linsky et al., 1979; Hall, 1996), but such methods are unlikely to achieve better than about 15$20 \%$ accuracy. The $S$-flux calibration, using flux-calibrated spectra rather than the empirical flux scale approach of Linsky et al. (1979) and others, has been examined by Cincunegui et al. (2007); while the available flux-calibrated data are limited, this seems the more desirable approach.

\subsection{The Vaughan-Preston Gap and the evolution of stellar dynamos}

The overall distribution of chromospheric HK fluxes in the MWO sample also revealed an interesting bifurcation in the activity level, shown in Figure 12 (Vaughan and Preston, 1980) and now known as the Vaughan-Preston Gap. Vaughan and Preston (1980) speculated that this could result either from a fundamental property of dynamo evolution in cool stars, or a statistical artifact.

The MWO time series clearly show that the young, high-activity stars exhibit predominantly irregular cycles, while older, Sun-like stars (i.e., those below the gap) have more well-defined cycles (Vaughan, 1980; Baliunas et al., 1995). Durney et al. (1981) argued that this was a manifestation of a rapid change in dynamo number from large, multiple-mode values to small, single mode values, without requiring any sudden spindown of the star. Some subsequent studies argued against that interpretation (Noyes et al., 1984), or suggested it was merely a statistical effect created by intrinsic upper and lower bounds of the $S$ index arising from photospheric background and chromospheric saturation (Hartmann et al., 1984), but the gap appears in surveys employing both $R^{\prime}$ (Henry et al., 1996; Gray et al., 2006) and absolute flux (Hall et al., 2007b) and does appear to be a real aspect of chromospheric activity in Sun-like stars.

The twin issues of whether the Vaughan-Preston gap is real, and if so, what it implies for the evolution of the magnetic dynamo that drives chromospheric activity, have been fruitfully studied in terms of quantities like the Rossby number $\mathrm{Ro}=P_{\mathrm{rot}} / \tau_{c}$ (the ratio of the rotation period to the convective turnover time, e.g., Noyes et al., 1984), or the ratio of cycle and rotation periods $P_{\text {cyc }} / P_{\text {rot }}$ (e.g., Soon et al., 1993). Brandenburg et al. (1998) and Saar and Brandenburg (1999) explored relationships between the ratio of the cycle and rotational periods and $R_{\mathrm{HK}}^{\prime}$, finding that most MWO stars fall in two distinct parallel samples which they called the active (A) and inactive (I) branches, suggesting that a rapid increase in $P_{\text {rot }} / P_{\text {cyc }}$ by about a factor of 6 occurs at $\approx 2-3$ Gyr, switching stars from the A to the I branch. Böhm-Vitense (2007) has suggested

Living Reviews in Solar Physics

http://www. livingreviews . org/lrsp-2008-2 

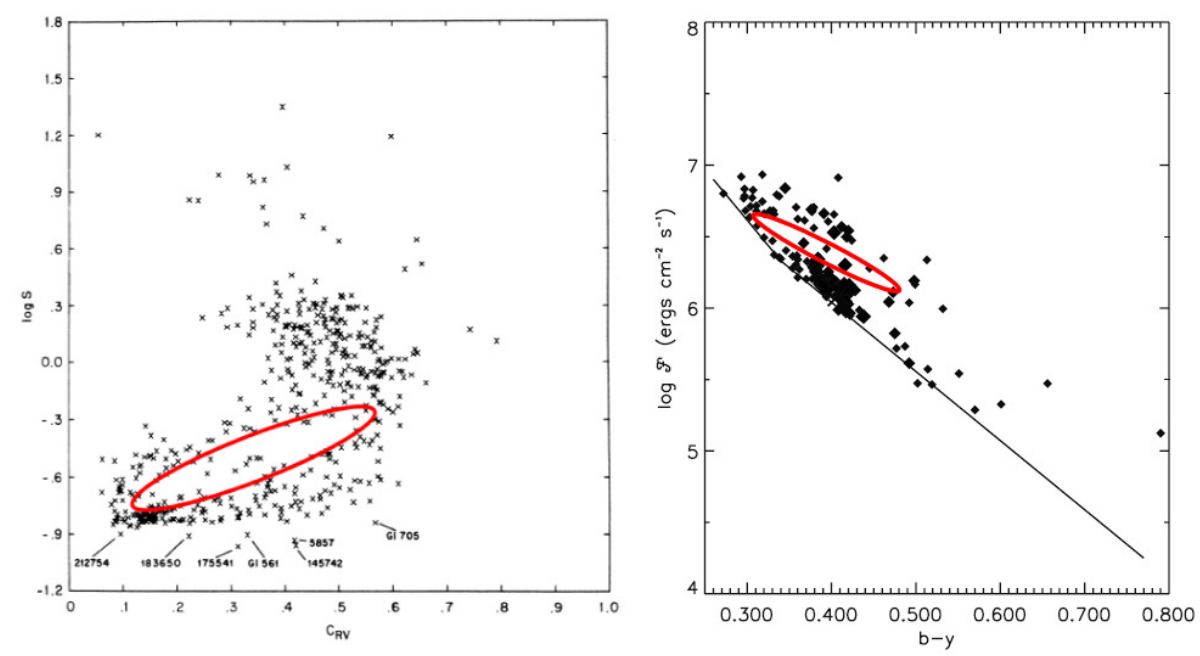

Figure 12: The so-called "Vaughan-Preston Gap," indicated in the figure with red ellipses, is a relative absence of $\mathrm{F}$ and $\mathrm{G}$ stars of intermediate activity levels. In addition to appearing in the large surveys by Henry et al. (1996) and Gray et al. (2006), it is present in the activity means obtained by the synoptic programs at MWO (left panel; $C_{\mathrm{RV}}$ is the Mount Wilson instrumental color index) and Lowell (right panel) (adapted from Vaughan and Preston, 1980 and Hall et al., 2007b).

that this means different dynamos operate at different times in a star's life, since I-branch stars of given mass require many more rotations to generate an activity cycle than A-branch stars; she also notes that the Sun lies squarely between the A and I branches (see also Section 5).

\subsection{Basal heating of the chromosphere}

A color-flux diagram of the Mount Wilson stellar sample reveals a clear, color-dependent lower limit to the CaII H \& K flux. A lower limit here is not surprising, since the triangular bandpass of the MWO photometer admits light from the photospheric line wings unrelated to the chromospheric activity; however, IUE observations of the Mg II h \& k fluxes for 30 of Wilson's original survey stars revealed the same lower limit (Doherty, 1985), suggesting the presence of a non-radiative equilibrium, non-cycle related source of heating. Schrijver (1987) showed that log linear power law relations between the flux densities in various chromospheric, transition region, and coronal line fluxes were significantly tightened by the removal of a color and luminosity class-dependent basal flux $\phi_{i}$ from each set of fluxes, e.g., $\log \left(\mathcal{F}_{i}-\phi_{i}\right)=a \log \left(\mathcal{F}_{j}-\phi_{j}\right)+b$. Since the basal fluxes were entirely uncorrelated with coronal (i.e., magnetic) emission and agreed well with model-predicted fluxes in field-free regions of the atmosphere, Schrijver (1987) argued that the basal flux was of acoustic origin.

A detailed examination of the various sources of the flux in the MWO instrumental bandpass confirmed that stars have "basal chromospheres" responsible for at least part of the observed activity (Schrijver et al., 1989b). Solar observations showed that the Sun's basal emission was as inhomogeneous as its magnetic activity, with basal intensities being observed in field-free regions near the centers of supergranules (Schrijver, 1992); this also agreed with the basal emission's being of acoustic rather than magnetic origin. The acoustic explanation for heating of the non-magnetic chromosphere was until recently considered the most viable (e.g., Schrijver, 1995; Buchholz et al., 1998; Fawzy et al., 2002), though Schrijver (1995) noted in his review of the subject that weak turbulent magnetic fields "could not be ruled out entirely".

Judge et al. (2003) have argued that this last caveat may be the case: based on hydrodynamic 
simulations of the chromospheric Ca II $\lambda 1335$ multiplet, they conclude that the observed basal emission, at least in this line, is magnetic in origin, possibly arising from weak internetwork fields such as observed by Lites (2002). Using a specially uniformly timed sequence of Transition Region and Coronal Explorer (TRACE) observations, Fossum and Carlsson (2005, 2006) measured the acoustic flux in the solar chromosphere and concluded that the high frequency acoustic flux is a full order of magnitude less than the net radiative chromospheric losses, and that activity of the middle and upper chromosphere is dominated by the magnetic field. This has led to the idea of magnetoacoustic portals (Jefferies et al., 2006) allowing propagation of low-frequency magnetoacoustic waves via "leakage" through inclined flux tubes into the lower chromosphere. This interpretation also fits naturally with the formation of spicules via photospheric p-mode shocks propagating upward through flux tubes, driving the formation of the relatively short-lived spicules (de Pontieu et al., 2004).

The most likely scenario at present accommodates both acoustic and small-scale magnetic mechanisms. As noted above, ample evidence for acoustic contributions has emerged, but a "multiscale magnetic carpet" (Schrijver and Title, 2003) of surface fields unrelated to the dynamo is strongly suggested by the recent, high-resolution solar observations. This latter component will have important implications when we consider the nature of Maunder Minimum stars in Section 4.8.

\subsection{Synoptic space-based observations}

The high temperature of outer stellar atmospheres means that most useful spectral diagnostics will lie in the UV beyond the ozone cutoff, EUV, and X-ray portions of the spectrum. The initial rocket and satellite observations of the late 1960s and early 1970s confirmed the existence of these indicators of chromospheric, transition region, and coronal activity in the solar UV spectrum (Goldberg et al., 1968) and for a few bright stars (e.g., McClintock et al., 1975; Evans et al., 1975; Dupree, 1975). The launch of IUE opened the spectrum from $\lambda \lambda 1150-3300 \AA$ to near-continuous observation; likewise, the HEAO, ROSAT, and XMM-Newton X-ray observatories have allowed investigation of the evolution of stellar coronal activity unobservable from the ground.

The priorities and scheduling of these observatories has largely precluded extensive synoptic observing, but we are nevertheless beginning to develop a multiwavelength picture of long-term stellar variations. Hempelmann et al. (1996) compared MWO HK data with ROSAT survey and pointed observations of a large set of Sun-like stars and found that the three broad types of stellar variability in the MWO sample (see Figure 9) corresponded to distinct levels of X-ray emission, with irregular stars having the highest X-ray fluxes and flat activity stars the lowest. While this is not unexpected, it does create an additional method of checking for cycle minima versus true Maunder Minimum states (cf. Section 4.8). From statistical arguments, Hempelmann et al. (1996) also showed that we should expect to see coronal cycles in Sun-like stars if a sufficient X-ray time series could be gathered.

This proposition has been borne out by more recent work. A synoptic observing program of the binary HD 81809 with XMM-Newton begun in 2001 shows evidence for a coronal activity cycle; interestingly, the cycle appeared to be shifted in phase by about one year from the HK data (Favata et al., 2004). In contrast, Hempelmann et al. (2006) have identified a clear coronal activity cycle, using XMM-Newton observations combined with earlier ROSAT observations, in 61 Cygni A = HD 201091 (see Figure 13). The cycle is only $1 / 3$ the amplitude of the solar coronal cycle variation, although 61 Cyg A has a much higher $S$ index than the Sun, and unlike HD 81809, the chromospheric and coronal cycles are tightly in phase.

These results provide a fascinating first look at the link between chromospheric and coronal cyclic activity, although the observational perspective is where Olin Wilson was in about 1970. The first two good results show us coronal cycles both in phase and offset with the chromospheric emission, and synoptic observations of additional stars will no doubt turn up additional surprises.

Living Reviews in Solar Physics

http://www. livingreviews.org/lrsp-2008-2 


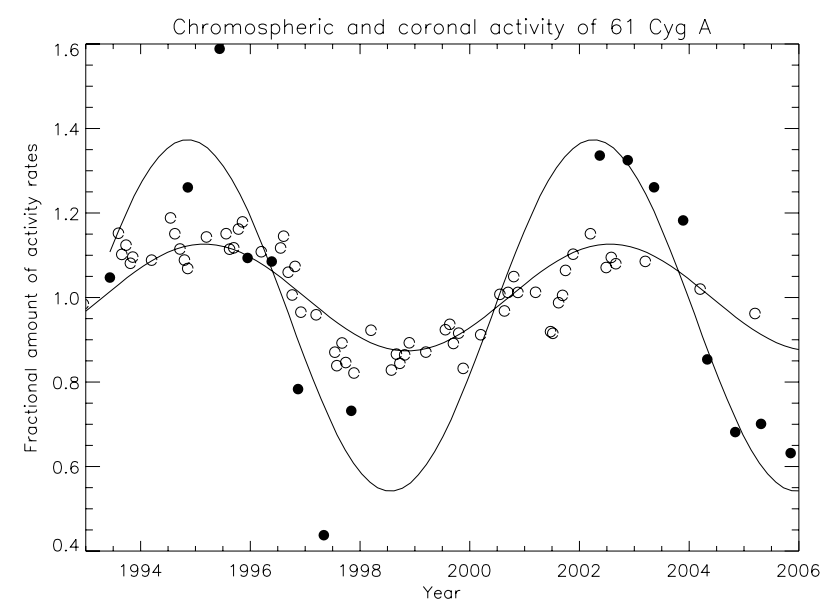

Figure 13: Figure 8 from Hempelmann et al. (2006), the first and clearest long-term observation of coherent chromospheric and coronal activity. The relative variability of the star is shown for the ROSAT and XMM-Newton observations (filled circles) and the combined Mount Wilson and Lowell HK series (open dots).

\subsection{Sun-as-a-star observations}

High resolution observations of the Sun provide a crucial conceptual and theoretical basis for interpreting stellar activity, but unresolved, "Sun-as-a-star" observations are essential for comparing solar activity directly with the unresolved stellar proxies. Ground-based observations of chromospheric and photospheric proxies since 1974 from the National Solar Observatory (NSO) at Kitt Peak (KP) and Sacramento Peak (SP) are reported in a number of papers (e.g., White and Livingston, 1981; Livingston and Holweger, 1982; Keil and Worden, 1984; Worden et al., 1998, and references therein), and Livingston et al. (2006) have provided a thorough summary of these synoptic observations from 1974 through 2006.

Figure 14 shows representative long-term solar observations from the NSO program (from Livingston et al., 2006, Figure 18). The roughly 25\% variation in Ca K is apparent; identical Ca K records are obtained via lunar observations from Mount Wilson (Baliunas et al., 1995) and direct solar observations at Lowell (Hall et al., 2007b). More surprising (and unexplained) is that the central intensities of the photospheric lines deepened at the 1986 minimum but failed to do so for the 1996 minimum (see center data series of Figure 14). This is particularly unexpected in light of evidence that line blanketing rather than continuum variations are predominantly responsible for cycle-timescale irradiance variations (Mitchell Jr and Livingston, 1991; Unruh et al., 1999). Livingston et al. (2006) suggest that there is a hint of a 22-year cycle in the data, but that is clearly an issue that further observation must decide.

\subsection{Chromospheric activity and brightness variations}

As discussed in Section 3.5, stellar chromospheric activity is related to the star's magnetic structure and therefore to the presence of photospheric features, such as spots and faculae, that modulate luminosity. Nimbus-7 began the first long-term observations of the total solar irradiance (TSI), initiating a data set now of critical importance in assessing the relationship between chromospheric activity and solar and stellar luminosity. Radiometers aboard Nimbus-7 (launched in 1978) and the Solar Maximum Mission (1980) revealed that the solar "constant" was variable in a manner that appeared directly correlated with the activity level (e.g., Fröhlich, 1987, see Figure 4), consistent 


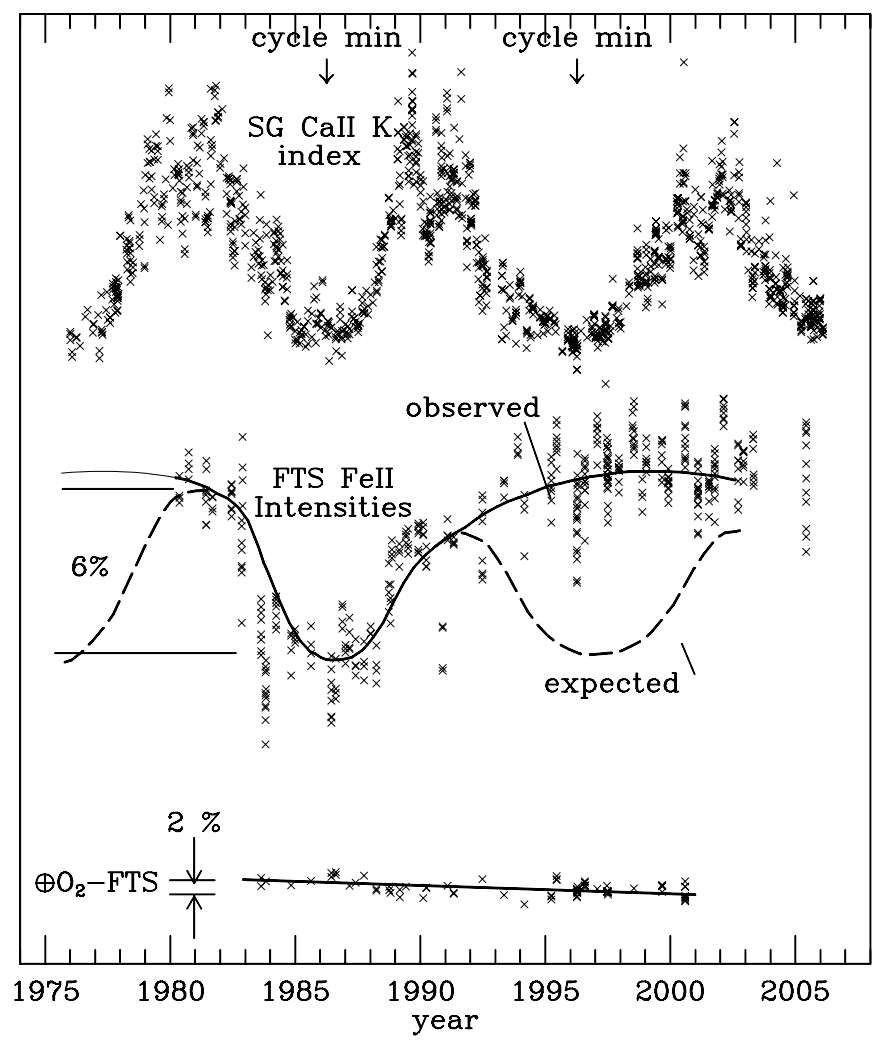

Figure 14: Figure 18 from Livingston et al. (2006). Top: the Ca K record, showing the direct correlation with the magnetic activity cycle. The scale is not shown in this figure, but the amplitude of the cycle in $\mathrm{Ca} \mathrm{K}$ is about $25 \%$. Center: the record for five photospheric iron lines, showing the lack of response of the lines to the 1996 Cycle 22/23 minimum. Bottom: control series, an $\mathrm{O}_{2}$ line normalized to air mass 1.0. 
with expectations that modulation of facular area by magnetic fields should lead to luminosity variations (Foukal and Vernazza, 1979). Models of solar irradiance variability typically apply a two-component model of facular brightening and sunspot darkening to reproduce the $\approx 0.1 \%$ cyclic variability in the solar irradiance record (Chapman, 1987; Foukal and Lean, 1990), although disagreement persists about the composite irradiance record and the presence or absence of a secular increase in brightness from the cycle minimum of 1986 to that of 1996 (Willson, 1997; Fröhlich and Lean, 1998). Non-facular sources of brightness changes have been postulated (Kuhn and Libbrecht, 1991), but Lean et al. (1998) find that magnetic sources, both from active regions and the active network, can explain the observed solar output variations, and recent work has employed sunspotfaculae-network models to satisfactorily reproduce irradiance variations (Krivova et al., 2007).

The relationship between solar luminosity and chromospheric activity has spurred complementary stellar studies, the most extensive of which combined the Mount Wilson HK activity observations with 11 years of Strömgren $b$ and $y$ photometry taken at Lowell Observatory of a sample of Sun-like stars (Lockwood et al., 1997; Radick et al., 1998). The essential results appear in Figure 15. The two panels at left are color-activity plots, and Radick et al. (1998) found that on cycle timescales, more active (and hence younger) stars with $\log R_{\mathrm{HK}}^{\prime}<-4.7$ display, with few exceptions, inverse correlations of chromospheric activity and brightness, while older (including solar-age) stars displayed direct activity-brightness correlations (as of course does the Sun itself). An initial interpretation is that stars switch from spot-dominated to facular-dominated brightness variations at $\approx 2$ Gyr. Equally interesting are the activity-variability plots on the right side of Figure 15; the Sun has a vigorous chromospheric cycle relative to its closest stellar analogs (upper right) but is photometrically sedate (lower right). Radick et al. (1998) found that this discrepancy could not be due to inclination effects (i.e., that a sample of randomly inclined stars might appear more photometrically variable than the Sun), a result corroborated by Knaack et al. (2001).

The Lowell photometric program ended in 1998, but observations of an expanded Sun-like star sample continued at the Fairborn Observatory, using high-precision robotic photometric telescopes, in southern Arizona (Henry, 1999). Observations from this program have been combined with the Lowell photometry and the MWO data to provide a 20-year examination of chromospheric activity and brightness variations for bright solar analogs (Lockwood et al., 2007). The conclusions from this study are not significantly different from those of Radick et al. (1998) (a point I shall return to in Section 5), but the photometric quiescence of the Sun remains a nagging issue. Lockwood et al. (2007) point out that the Sun's apparently low photometric variability may simply stem from their small sample size, but also that the issue bears further investigation. Along those lines, the star widely considered to be an excellent solar twin, 18 Sco = HD 146233 (Porto de Mello and da Silva, 1997), has been found to have chromospheric activity similar to or slightly exceeding that of the Sun, a Sun-like cycle amplitude (though of somewhat shorter length) and similarly low photometric variability of about $0.09 \%$ over a full activity cycle (see Figure 16, from Hall et al. (2007a)). Whether this similarity exists for other solar twins obviously remains to be seen. Recently, Meléndez and Ramírez (2007) have found that the star HIP 56948 is a better solar twin than $18 \mathrm{Sco}$, on the basis of its lower $\mathrm{Li}$ abundance. This is a good indication that a larger sample of good solar analogs and twins, as we would statistically expect, will emerge once surveys are pushed to fainter magnitudes than the MWO and Lowell programs have been able to achieve.

\subsection{Flat activity stars and the Maunder Minimum}

An intriguing aspect of chromospheric variability has been its absence in a number of Sun-like stars, including the Sun itself. Eddy (1976) created renewed interest in the period of solar inactivity from 1645-1715 which he called the Maunder Minimum, after the British astronomer who first noted it in the 1890s, during which the solar activity cycle did not shut down entirely, but operated at a greatly reduced level (Beer et al., 1998; Ribes and Nesme-Ribes, 1993). The solar activity-irradiance 

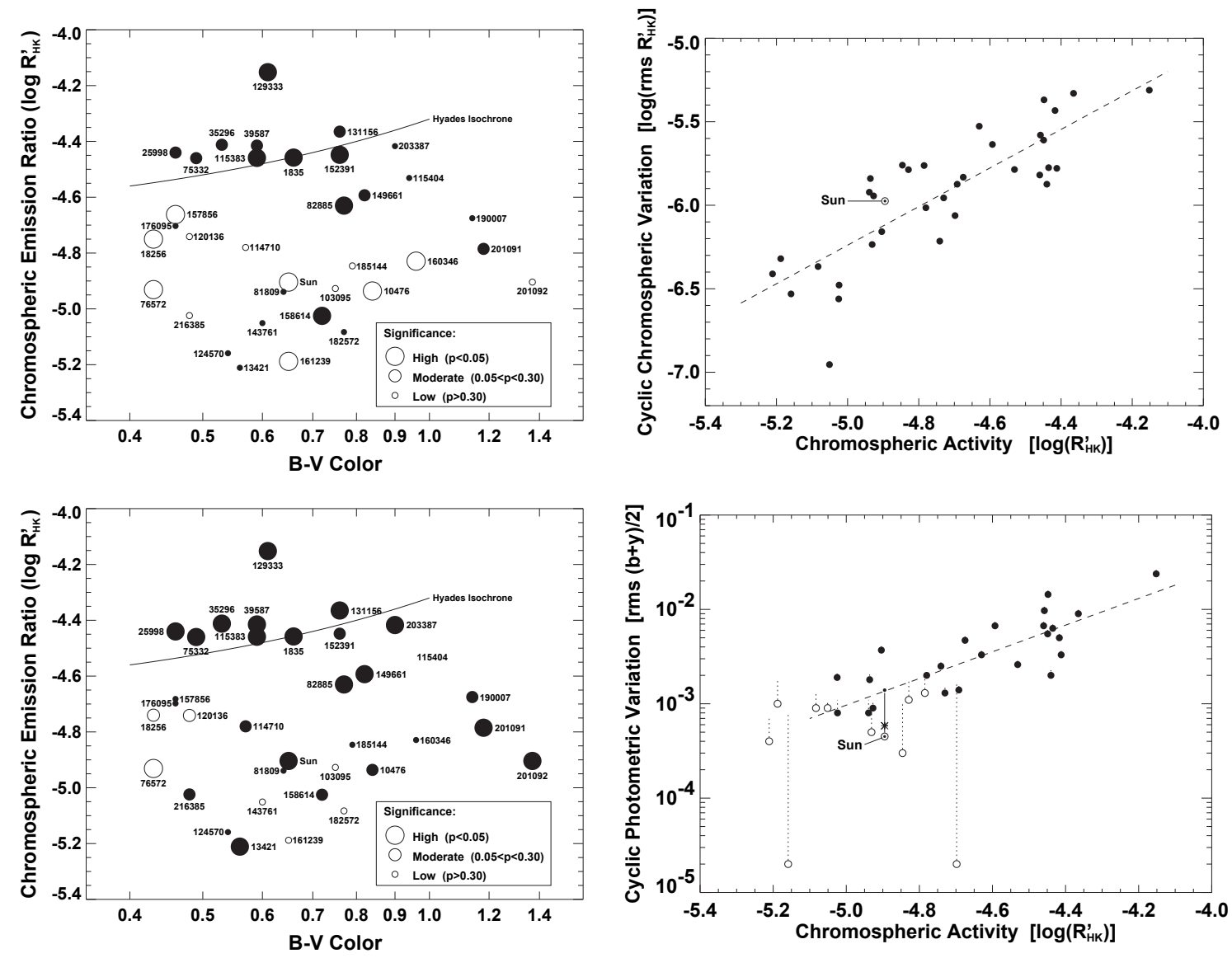

Figure 15: Left panels: long (top) and short (bottom) term brightness variations as a function of activity level and color. Filled circles indicate inverse correlations of brightness and HK emission; open circles indicate direct correlations. Right panels: HK variability (top) and photometric variability (bottom) versus overall activity level. Relative to comparable stars, the Sun has a relatively strong chromospheric cycle but low photometric variability (from Radick et al., 1998). 

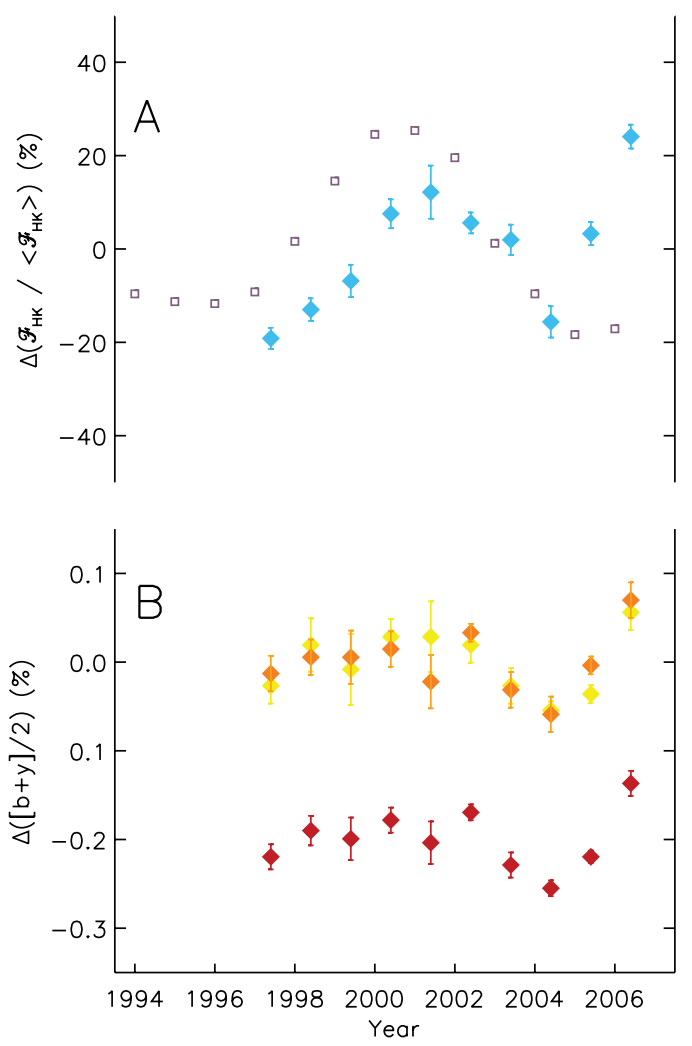

Figure 16: The brightness variations and chromospheric activity of the solar twin 18 Scorpii over 10 years. At top are the Lowell H \& K seasonal means (blue diamonds), along with the solar seasonal means for comparison (purple squares). The 18 Sco photometric means are shown at bottom, relative to individual comparison stars (yellow and orange) and the weighted mean (red) (from Hall et al., 2007a). 
correlations described in Section 4.7 raised interest even further in so-called "grand minima", especially in regard to estimating solar contributions to terrestrial climate change. Creation of solar irradiance reconstructions has proceeded along two fronts: (i) modeling cycle-timescale irradiance fluctuations via the cyclic evolution of bright faculae, active network and plage, and dark sunspots, and (ii) examining possible secular changes in the minimum activity level via evolution of the quiet network. I concentrate on point (ii) here.

A key initial problem was to estimate the Sun's brightness in the absence of a quiet network. Mount Wilson observations of the $S$ indices of selected non-cycling stars suggested their Ca II H \& K emission lay well below that of the contemporary solar minimum (Baliunas and Jastrow, 1990), from which White et al. (1992) deduced that the K emission from the Sun during the Maunder Minimum may have been as little as $11 \%$ that of current cycle minima. On this basis, Lean et al. (1992) estimated that the Sun may have been $0.24 \%$ fainter during the Maunder Minimum, a figure that guided subsequent irradiance reconstructions that included both cyclic and secular components (e.g., Lean et al., 1995). Further examination of the activity records and $S$ values of flat activity stars yielded estimates of long-term solar variability as much as $0.4 \%$ (Baliunas and Soon, 1995) or $0.6 \%$ (Zhang et al., 1994). However, Hall and Lockwood (2004) were unable to recover the bimodal cycling versus non-cycling star activity distribution of Baliunas and Jastrow (1990) using a larger sample, and Wright (2004) argues that previously identified Maunder Minimum stars are actually evolved. Recent irradiance reconstructions have employed small secular components to the solar activity trend since 1715 (e.g., Wang et al., 2005; Krivova et al., 2007).

Renewed efforts to identify Maunder Minimum candidates demonstrably on the main-sequence have been undertaken by Judge et al. (2004) and Judge and Saar (2007). Two leading candidates are $\tau$ Ceti $=$ HD 10700 and HD 143761, and Judge and Saar (2007) used UV and X-ray observations of these stars to detect the presence of transition region and coronal emission, concluding that magnetic activity for at least these two flat activity stars is not significantly reduced below a cycle minimum state. We therefore presently have a tentative view of flat activity stars as having smallscale magnetic fields and, technically, chromospheric activity, while lacking the large-scale active region fields characteristic of cycling stars. The sample on which this is based remains small, and continued studies of low-activity stars will help clarify matters. The Holy Grail in this area is a convincing observation of a star entering or leaving a Maunder Minimum; the star HD 3651 in the MWO program may have done this, and the star $\psi$ Ser $=$ HD 140538 makes an excellent case for the end of a Maunder Minimum state in the Lowell data (see Figure 17). Essential adjunct observations, of course, are the luminosity variations of such stars as their activity characteristics change.
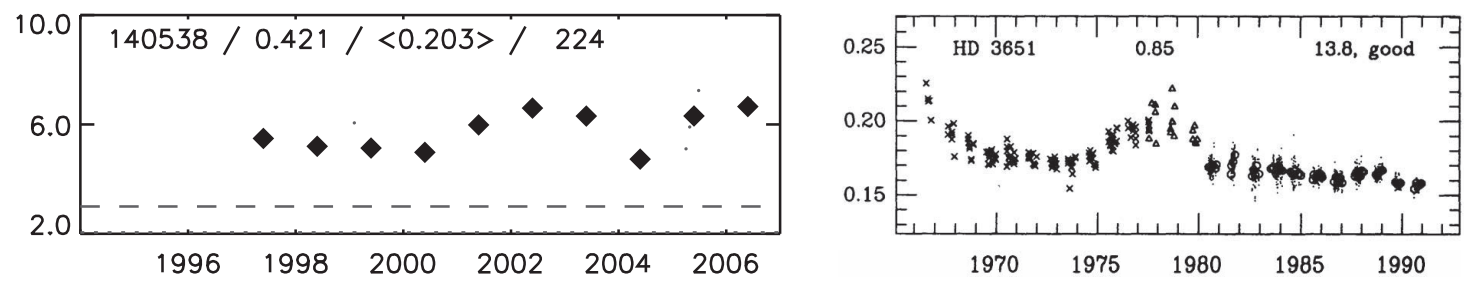

Figure 17: Left: HD 140538 appears to have made a transition from a flat activity state to an unusually short cycle in 2000 (from Hall et al., 2007b). Right: HD 3651 shows evidence of having entered a flat activity state around 1980 (from Baliunas et al., 1995). 


\subsection{Going further}

Section 4.1: An unavoidable aspect of all the stellar time series is their uneven sampling, which manifests itself both on yearly (due to the stars' observing seasons) and intraseason (due to telescope scheduling and weather vagaries) timescales. A widely used technique for handling the MWO data is an adaption of the Lomb-Scargle periodogram to unevenly sampled data described by Horne and Baliunas (1986). More recently, Frick et al. (1997) have developed an improved technique, using a similar extension to standard wavelet analysis.

I must also note in passing that the intentional focus of this review on Sun-like stars has completely ignored an important set of the most chromospherically active stars we know of: close binaries with cool components. The fundamental introduction in the modern literature is Hall (1976), introducing, among others, the archetypal active close binaries, the RS CVn systems, which typically include a $\mathrm{G}$ or $\mathrm{K}$ subgiant and a late $\mathrm{F}$ or $\mathrm{G}$ dwarf with orbital periods from a few days to a few weeks. Since such systems typically are synchronous, both components are greatly spun up and exhibit greatly enhanced chromospheric emission relative to field stars. They are thus excellent test cases for exploring the limits of activity as the atmospheres approach saturation, as well as Doppler imaging techniques (e.g., Vogt et al., 1987; Strassmeier, 1996) to map the rough distribution of surface features. The reader may find numerous texts on the subject (e.g., Hilditch, 2001), and the recent literature contains a number of surveys and catalogs of the brighter systems (Strassmeier et al., 1993; Montes et al., 2000).

Section 4.2: Interpreting the ground-based observations of stellar chromospheric activity in terms of absolute flux has caused, as J.R.R. Tolkien put it, "a gorgeous row". The Mount Wilson $S$ index itself is in reasonably good shape; the various other programs that have either replicated $S$ or created $S$-like quantities are in reasonable agreement (e.g., Hall and Lockwood, 1995; Gray et al., 2003; Wright et al., 2004; Hall et al., 2007b), despite its shortcomings for physical interpretation. However, fine points in color corrections and flux calibration have led to discrepant formulations of $\log R_{\mathrm{HK}}^{\prime}$ and absolute surface flux from a given $S$ measurement, as well as difficulty reconciling solar and stellar observations (Noyes et al., 1984; Rutten, 1984; Schrijver et al., 1989a). Duncan et al. (1991) wrote that "in general the agreement [of $S$ /flux calibrations] is good", though the continued ruffling of feathers in the literature suggests this assessment was generous. Hall et al. (2007b) have revisited the issue, and while the $S$ /flux and solar/stellar calibrations seem to be in good agreement, the empirical determination of fluxes still has distressingly large errors of $15-$ $25 \%$, stemming largely from uncertainties in the quantities needed to derive flux scales in the manner of Linsky et al. (1979). The uncertainties are aggravated for low-activity stars that are currently of particular interest (see Section 4.8), since most of the flux in the typical measurement bandpasses does not arise from dynamo-related activity and must be removed to obtain $R^{\prime}$ (see Noyes et al., 1984) or the excess flux $\mathcal{F}_{\mathrm{HK}}$ (see Schrijver et al., 1989b; Hall et al., 2007b). Using carefully flux-calibrated spectra as a starting point (Cincunegui et al., 2007) may be a promising approach to resolving some of these issues. Beyond all these issues, the flux-calibrated space-based data also require careful treatment; for a thorough discussion, see Judge (1990).

Section 4.6: Not surprisingly, the NSO HK records correlate well with other measures of the Sun's overall level of activity, such as the $10.7 \mathrm{~cm}$ flux and the sunspot number. The latter, because of its 400-year baseline, is fundamental to the irradiance reconstructions described briefly in Section 4.7, and has also led to extensive work on an ancillary topic, the prediction of solar activity via application of various dynamo, statistical, and in some cases numerological models (for a review see Hathaway et al., 1999) to replicate the observed sunspot records. Predictions for solar cycle 24, which should last from ca. 2008-2017, indicate that it should be either very weak (Schatten, 2005; Penn and Livingston, 2006), fairly moderate (Sello, 2003), or extremely strong (Dikpati et al., 2006). I hazard that one of these is correct. More seriously, the Sun will answer the question for us in due course, and will provide a useful check on models that lead to these 
divergent predictions, and perhaps further insight on the claim by Bushby and Tobias (2007) that the whole enterprise is futile.

Section 4.7: Another long-suspected source of variations in solar irradiance are secular changes in the Sun's radius. Some evidence suggested such changes happened in the past (Gilliland, 1981) as well as over recent solar cycles (Noël, 2004), but more recent observations from space find no evidence for solar radius changes (Kuhn et al., 2004). Like Abbott's early attempts to measure the solar constant, this appears to be another in the long list of examples of observations phenomenally difficult to do from the ground.

Section 4.8: An interesting aspect of recent solar and stellar activity research is the strong evidence for a fingerprint of solar activity in terrestrial climate records (the subject of a great many reviews; as a fairly recent starting point, see Haigh (2001), Rind (2002), or the recent lectures by M. Lockwood, J. Haigh, and M. Giampapa on the Sun, solar analogs, and climate (Haigh et al., 2005). The possibility that grand minima (or maxima) may induce luminosity excursions beyond those of the modern solar cycle makes the puzzle all the more interesting. A full discussion of this issue is far out of the scope of this review. The one-sentence summary is as follows: simple and dominant correlations between solar activity and climate (Friis-Christensen and Lassen, 1991) generally appear to be untenable (Damon and Peristykh, 1999), and although the Sun appears to be in an unusually high-activity state relative to the past several millennia (Solanki et al., 2004), the observed modern irradiance variations and likely secular evolution since the Maunder Minimum are insufficient to be the dominant contributor to late 20th century global warming (Wang et al., 2005; Foukal et al., 2006). However, it is clear that the Sun influences climate, likely in ways we do not fully appreciate. Blanket dismissals of solar variations in assessing modern climate change sometimes border, in my opinion, on the overconfidence that is damaging to healthy scientific skepticism, and continued investigation of the important problems is entirely warranted. For an excellent recent review of solar influences on climate and the outstanding issues, I refer the reader to Haigh (2007). Recent books and monographs covering the Sun-Earth connection in detail are Hoyt and Schatten (1997), Friis-Christensen et al. (2000), Benestad (2002), and Pap and Fox (2003). 


\section{A View to a Chromosphere}

Toward the end of the 1972 conference on stellar chromospheres (Jordan and Avrett, 1973), Olin Wilson said: "It is important to realize that a chromosphere is a completely negligible part of a star. Neither its mass nor its own radiation makes a significant contribution to those quantities for the star as a whole". If Wilson was intending to be provocative, he succeeded magnificently, drawing a lengthy rebuttal and summation from R.N. Thomas. While the latter part of Wilson's statement may be technically correct, we now know that the chromosphere is directly physically associated with structures that $d o$ contribute to the properties of the star as a whole, especially at high energies where ionizing radiation can have a profound influence on Earth and the heliosphere. With the significance of the chromosphere well established, let us examine a few threads in the previous sections with an eye toward future work.

What is the next generation of synoptic observations? Through 40 years of effort, Olin Wilson, Sallie Baliunas, and their colleagues created one of the outstanding data sets of modern astrophysics, but despite this, our view on long-term stellar chromospheric variability has a number of critical limitations. For example, it is important to realize that Ca II $\mathrm{H} \& \mathrm{~K}$ is a completely negligible part of the spectrum. In his defense of the CO-mosphere, Ayres (2002) cautions us not to overlook "spectral backwaters" such as the far infrared. We would do well to note that the brackish water reaches our doorstep. Probably the critical region to sample from the ground in future synoptic studies is $\lambda \lambda 4000-5000$, which includes in particular the CH G band near $\lambda 4300$, which precisely tracks magnetic flux concentrations directly related to the chromospheric and coronal activity of the star (Schüssler et al., 2003). The Lowell SSS is equipped with an echelle that samples $\approx \lambda \lambda 5100-9000$, primarily useful in surveying the chromospheric proxies in that part of the spectrum, but suffering, particularly redward of $\lambda 6000$, from numerous telluric blends. The solar cycle and even rotational signal is visible in the myriad "proxies" in the optical spectrum (Livingston et al., 2006; Livingston and Holweger, 1982; Hall and Lockwood, 1998), and these regions provide much more light than the dark heart of the Ca II lines. This is particularly important if we extend our observations to fainter solar analogs (as with the Giampapa et al., 2006 Hydra observations of Ca II, done with no small difficulty at $m_{V} \approx 14$, to study the distribution of activity in M67). Given the increasing realization of the importance of the evolution and distribution of the small-scale magnetic fields, the ideal next-generation synoptic instrument will ideally survey $\lambda \lambda 3900-6600$.

What is the future of $\mathbf{H} \& \mathbf{K}$ ? I should be as provocative as Wilson if I said it was time to send Ca II H \& K to a home for retired proxies, but I do believe that if a research "cusp" such as we saw circa 1960, 1980, and 2000 is to occur in the future, it will not involve synoptic H \& K observations. There are still insights to be gleaned; the Lowell observations are satisfactorily stitched to the MWO series and are now extending many of them, and we still have points to make about cycle amplitude evolution and secular evolution of activity at cycle minima. However, the recent spectroscopic-photometric retrospective published by Lockwood et al. (2007) is largely incremental over the previous reports from that program (Lockwood et al., 1997; Radick et al., 1998); and while the SSS HK database (Hall et al., 2007b) offers time series and some interesting cycle detections beyond those of Baliunas et al. (1995), the overall behavior of the target set yields few surprises. The essential astrophysical utility of long-term observations of activity is the insights about the dynamo discussed in Section 4.3; further advances will come from (1) broadening the set of synoptic ground-based proxies and (2) ensuring they are obtained with contemporaneous photometry (cf. Radick et al., 1998; Hall et al., 2007a) and, if at all possible, EUV and X-ray observations as Favata et al. (2004) and Hempelmann et al. (2006) have begun.

How special is the Sun? Although this is a commentary on stellar chromospheric activity, the field has become so intertwined with solar work, and the evidence that stellar variations arise from structures analogous to solar phenomena so overwhelming, that it is no longer possible to 
study magnetic phenomena in either the Sun or its cousins in isolation. I have noted throughout that the recent detailed views of the Sun are important guides for stellar work, assuming that the Sun is a typical G star. In a gross sense, it appears to be, but if the devil is in the proverbial details, is it really? Solar twins have been hard to come by, though the well-studied sample is severely magnitude-limited to perhaps $m_{V}=7.5$. The "Top Ten" solar analogs of Soubiran and Triaud (2004) include the one star everyone seems to agree on, 18 Scorpii = HD 146233 as \#1; the rest are variously poorly studied, possibly evolved (HD 95128), old (HD 186427), or binaries (HD 10307). Only very recently are other genuine twins beginning to be found (Meléndez and Ramírez, 2007). One essential issue here is the still puzzling photometric quiescence of the Sun relative to stars of similar color (Lockwood et al., 2007; Radick et al., 1998). Interestingly, the photometric variability of the one solar twin for which it has been measured, $18 \mathrm{Sco}$, is nearly identical to the Sun over a full activity cycle (Hall et al., 2007a) - and therefore also an outlier relative to most dwarfs with $B-V \approx 0.65$. So we can now pick our statistical poison: one star does not a trend make; or, if the one star agreed to be a solar twin is also a photometric outlier, is there something we don't know about the Sun? Clearly, ongoing, synoptic observations of the slowly growing sample of solar twins are critical, since it is otherwise difficult to approach this question without treading uncomfortably close to the anthropic principle. Böhm-Vitense (2007), however, in examining cycle lengths and rotation periods in the context of the "active" and "inactive" dynamo branches of Saar and Brandenburg (1999), notes that the Sun lies squarely between the two sequences and writes that "Clearly the Sun is not a good standard star for the discussion of stellar activity. (Is its special position between the sequences necessary to permit life on earth to evolve and survive?)". I believe Wilson would have enjoyed pondering this question. (With its shorter, $\approx 7.5$-year cycle, 18 Sco lies somewhat off the I branch though not discordantly so).

The next cusp. The arguments above show a profitable direction for future observations of stellar chromospheric activity most likely to lead to fundamental advances. The Sun is the one star for which we have an outstanding view to a chromosphere, and an increasingly detailed understanding of its fine structure. We also know the Sun spends some amount of its time in grand minima whose occurrence has been very recently argued to be chaotic (Usoskin et al., 2007). Wilson selected the MWO stars to sample a broad ensemble; we now need to contract the colormagnitude box around the Sun, push to fainter magnitudes (to at least $m_{V}=10$ ) to find other solar twins and analogs, and increase the time resolution of observations of stellar analogs of the modern and Maunder Minimum Sun. Essential complements will be contemporaneous highprecision photometry as Hall et al. (2007a) have done for 18 Sco, and at least a few EUV and X-ray observations per year as Hempelmann et al. (2006) are doing for 61 Cygni. Such work opens a host of questions: Is the Sun special? If so, how big is the parameter space that delineates a truly "Sun-like" dynamo and resulting activity? Are the Sun's grand minima similar to those of $\tau$ Ceti?

This is a modification of the question posed by Wilson three decades ago: Does the chromospheric activity of other Suns vary, and if so, how similarly to the Sun's? Hopefully, at some point, this Living Review can be updated with at least a partial answer. 


\section{Acknowledgments}

This work has been supported by grant ATM-0447159 to Lowell Observatory from the National Science Foundation. Writing this review was greatly facilitated by the resources available from NASA's Astrophysics Data System and the SIMBAD Astronomical Database, operated at CDS, Strasbourg, France. The author thanks Drs. Mark Giampapa and Tom Ayres for their time and thoughtfulness in reading a lengthy manuscript and returning excellent reviews with a number of useful suggestions that improved the final paper. 


\section{References}

Anderson, L.S., Athay, R.G., 1989, "Model Solar Chromosphere with Prescribed Heating", Astrophys. J., 346, 1010-1018. [DOI], [ADS] 3.7

Athay, R.G., 1970, "A Non-LTE Line-Blanketed Solar Model", Astrophys. J., 161, 713-735. [DOI], [ADS] 3.1

Audard, M., Osten, R.A., Brown, A., Briggs, K.R., Güdel, M., Hodges-Kluck, E., Gizis, J.E., 2007, "A Chandra X-ray detection of the L dwarf binary Kelu-1: Simultaneous Chandra and Very Large Array observations", Astron. Astrophys., 471, L63-L66. [DOI], [ADS] 2.3

Auer, L.H., Mihalas, D., 1969, "Non-LTE Model Atmospheres. I. Radiative Equilibrium Models with Lyman-Alpha", Astrophys. J., 156, 157-175. [DOI], [ADS] 2.2, 3.1

Ayres, T.R., 1979, "Chromospheric scaling laws, width-luminosity correlations, and the WilsonBappu effect", Astrophys. J., 228, 509-520. [DOI], [ADS] 3.2

Ayres, T.R., 2002, "Does the Sun Have a Full-Time COmosphere?", Astrophys. J., 575, 1104-1115. [DOI], [ADS] 3.6, 5

Ayres, T.R., Linsky, J.L., 1975, "Stellar Model Chromospheres. III. Arcturus (K2 III)", Astrophys. J., 200, 600-674. [DOI], [ADS] 3.4

Ayres, T.R., Linsky, J.L., Shine, R.A., 1974, "Stellar Model Chromospheres. II. Procyon (F5 IV-V)", Astrophys. J., 192, 93-107. [DOI], [ADS] 3.4

Ayres, T.R., Linsky, J.L., Rodgers, A.W., Kurucz, R.L., 1976, "Stellar Model Chromospheres. V. Alpha Centauri A (G2 V) and Alpha Centauri B (K1 V)", Astrophys. J., 210, 199-210. [DOI], [ADS] 3.4

Ayres, T.R., Marstad, N.C., Linsky, J.L., 1981, "Outer Atmospheres of Cool Stars. IX. A Survey of Ultraviolet Emission from F-K Dwarfs and Giants with IUE", Astrophys. J., 247, 545-559. [DOI], [ADS] 3.3

Ayres, T.R., Brown, A., Harper, G.M., 2003, "Buried Alive in the Coronal Graveyard", Astrophys. J., 598, 610-625. [DOI], [ADS] 2.3

Babcock, H.W., 1961, "The Topology of the Sun's Magnetic Field and the 22-Year Cycle", Astrophys. J., 133, 572-589. [DOI], [ADS] 2.2, 3.5

Baliunas, S.L., Jastrow, R., 1990, "Evidence for Long-Term Brightness Changes of Solar-Type Stars", Nature, 348, 520-523. [DOI], [ADS] 4.8

Baliunas, S.L., Soon, W.H., 1995, "Are Variations in the Length of the Activity Cycle Related to Changes in Brightness in Solar-Type Stars?", Astrophys. J., 450, 896-901. [DOI], [ADS] 4.1, 4.8

Baliunas, S.L., Horne, J.H., Porter, A., Duncan, D.K., Frazer, J., Lanning, H., Misch, A., Mueller, J., Noyes, R.W., Soyumer, D., Vaughan, A.H., Woodard, L., 1985, "Time-Series Measurements of Chromospheric Ca II H and K Emission in Cool Stars and the Search for Differential Rotation", Astrophys. J., 294, 310-325. [DOI], [ADS] 4.1

Baliunas, S.L., Donahue, R.A., Soon, W.H., Horne, J.H., Frazer, J., Woodard-Eklund, L., Bradford, M., Rao, L.M., Wilson, O.C., Zhang, Q., Bennett, W., Briggs, J., Carroll, S.M., Duncan, D.K., Figueroa, D., Lanning, H.H., Misch, T., Mueller, J., Noys, R.W., Poppe, D., Porter, A.C., Robinson, C.R., Russell, J., Shelton, J.C., Soyumer, T., Vaughan, A.H., Whitney, J.H., 1995,

Living Reviews in Solar Physics

http: //www. livingreviews.org/lrsp-2008-2 
"Chromospheric Variations in Main-Sequence Stars. II", Astrophys. J., 438, 269-287. [DOI], [ADS] $2.3,4.1,9,4.3,4.6,17,5$

Baliunas, S.L., Nesme-Ribes, E., Sokoloff, D., Soon, W.H., 1996, "A Dynamo Interpretation of Stellar Activity Cycles", Astrophys. J., 460, 848-854. [DOI], [ADS] 3.7

Baliunas, S.L., Donahue, R.A., Soon, W.H., Henry, G.W., 1998, "Activity Cycles in Lower Main Sequence and Post Main Sequence Stars: The HK Project", in Cool Stars, Stellar Systems, and the Sun, (Eds.) Donahue, R.A., Bookbinder, J.A., Proceedings of the Tenth Cambridge Workshop, held at Cambridge, Massachusetts, 15-19 July 1997, vol. 154 of ASP Conference Series, pp. 153-172, Astronomical Society of the Pacific, San Francisco. [ADS] 4.1

Beer, J., Tobias, S.M., Weiss, N.O., 1998, "An Active Sun Throughout the Maunder Minimum", Solar Phys., 181, 237-249. [DOI], [ADS] 4.8

Benestad, R.E., 2002, Solar Activity and Earth's Climate, Springer-Praxis Books in Environmental Science, Springer, Berlin; New York. [Google Books] 4.9

Biermann, L., 1948, "On the Cause of Chromospheric Turbulence and UV Excess of Solar Radiation", Z. Astrophys., 25, 161-177 2.2

Blanco, C., Catalano, S., Marilli, E., Rodonò, M., 1974, "Absolute Fluxes of K Chromospheric Emission in Main Sequence Stars", Astron. Astrophys., 33, 257-264. [ADS] 4.2

Blanco, C., Catalano, S., Marilli, E., 1976, "Absolute Fluxes of K Chromospheric Emission on the H-R Diagram", Astron. Astrophys., 48, 19-25. [ADS] 4.2

Bohlin, J.D., Vogel, S.N., Purcell, J.D., Sheeley Jr, N.R., Tousey, R., VanHoosier, M.E., 1975, "A Newly Observed Solar Feature: Macrospicules in He II 304 A", Astrophys. J. Lett., 197, L133-L135. [DOI], [ADS] 2.1

Böhm-Vitense, E., 2007, "Chromospheric Activity in G and K Main-Sequence Stars, and What it Tells Us About Stellar Dynamos", Astrophys. J., 657, 486-493. [DOI], [ADS] 4.3, 5

Brandenburg, A., Saar, S.H., Turpin, C.R., 1998, "Time Evolution of the Magnetic Activity Cycle Period", Astrophys. J. Lett., 498, L51-L54. [DOI], [ADS] 4.3

Buchholz, B., Ulmschneider, P., Cuntz, M., 1998, "Basal Heating in Main-Sequence Stars and Giants: Results from Monochromatic Acoustic Wave Models", Astrophys. J., 494, 700-714. [DOI], [ADS] 4.4

Bushby, P.J., Tobias, S.M., 2007, "On Predicting the Solar Cycle Using Mean-Field Models", Astrophys. J., 661, 1289-1296. [DOI], [ADS] 4.9

Carlsson, M., 2007, "Modeling the Solar Chromosphere", in The Physics of Chromospheric Plasmas, (Eds.) Heinzel, P., Dorotovic, I., Rutten, R.J., NSO Workshop No. 23, vol. 368 of ASP Conference Series, pp. 49-63, Astronomical Society of the Pacific, San Francisco 3.4, 3.7

Carlsson, M., Stein, R.F., 1995, "Does a Nonmagnetic Solar Chromosphere Exist?", Astrophys. J. Lett., 440, L29-L32. [DOI], [ADS] 3.6

Cayrel, R., 1963, "On a Purely Radiative Cause of Temperature Rise in a Stellar Atmosphere of Chromospheric Density", C. R. Acad. Sci., 257, 3309-3312 2.2

Cayrel de Strobel, G., 1996, "Stars Resembling the Sun", Astron. Astrophys. Rev., 7, 243-288. [DOI], [ADS] 4 
Chapman, G.A., 1987, "Solar Variability Due to Sunspots and Faculae", J. Geophys. Res., 92, 809-812. [DOI], [ADS] 4.7

Charbonneau, P., 2005, "Dynamo Models of the Solar Cycle", Living Rev. Solar Phys., 2, lrsp2005-2. URL (cited on 17 August 2007):

http://www.livingreviews.org/lrsp-2005-2 3.7

Cincunegui, C., Mauas, P.J.D., 2004, "Library of Flux-Calibrated Echelle Spectra of Southern Late-Type Dwarfs with Different Activity Levels", Astron. Astrophys., 414, 699-706. [DOI], [ADS] 4.1

Cincunegui, C., Díaz, R.F., Mauas, P.J.D., 2007, "H $\alpha$ and the Ca II H and K Lines as Activity Proxies for Late Type Stars", Astron. Astrophys., 469, 309-317. [DOI], [ADS] 4.2, 4.9

Damon, P.E., Peristykh, A.N., 1999, "Solar Cycle Length and 20th Century Northern Hemisphere Warming: Revisited", Geophys. Res. Lett., 26, 2469-2472. [DOI], [ADS] 4.9

de Pontieu, B., Erdély, R., James, S.P., 2004, "Solar Chromospheric Spicules from the Leakage of Photospheric Oscillations and Flows", Nature, 430, 536-539. [DOI], [ADS] 4.4

Dikpati, M., de Toma, G., Gilman, P.A., 2006, "Predicting the Strength of Solar Cycle 24 Using a Flux-Transport Dynamo-Based Tool", Geophys. Res. Lett., 33, L05 102. [DOI], [ADS] 4.9

Doherty, L.R., 1985, "A survey of Mg II h and k emission in near-solar-type stars", Mon. Not. R. Astron. Soc., 217, 41-53. [ADS] 4.4

Donahue, R.A., Baliunas, S.L., 1992, "Evidence of Differential Rotation in the Solar-Type Star HD 114710", Astrophys. J. Lett., 393, L63-L66. [DOI], [ADS] 4.1

Duncan, D.K., Vaughan, A.H., Wilson, O.C., Preston, G.W., Frazer, J., Lanning, H., Misch, A., Mueller, J., Soyumer, D., Woodard, L., Baliunas, S.L., Noyes, R.W., Hartmann, L.W., Porter, A., Zwaan, C., Middelkoop, F., Rutten, R.G.M., Mihalas, D., 1991, "Ca II H and K measurements made at Mount Wilson Observatory, 1966-1983", Astrophys. J. Suppl. Ser., 76, 383-430. [DOI], [ADS] 4.1, 4.9

Dupree, A.K., 1975, "Ultraviolet Observations of Alpha Aurigae from Copernicus", Astrophys. J. Lett., 200, L27-L31. [DOI], [ADS] 4.5

Durney, B.R., Mihalas, D., Robinson, R.D., 1981, "A Preliminary Interpretation of Stellar Chromospheric Ca II Emission Variations Within the Framework of Stellar Dynamo Theory", Publ. Astron. Soc. Pac., 93, 537-543. [DOI], [ADS] 4.3

Eberhard, G., Schwarzschild, K., 1913, "On the Reversal of the Calcium Lines H and K in Stellar Spectra", Astrophys. J., 38, 292-295. [DOI], [ADS] 3.2

Eddy, J.A., 1976, "The Maunder Minimum", Science, 192, 1189-1202. [DOI], [ADS] 4, 4.8

Evans, R.G., Jordan, C., Wilson, R., 1975, "Observations of Chromospheric and Coronal Emission Lines in F Stars", Mon. Not. R. Astron. Soc., 172, 585-602. [ADS] 4.5

Favata, F., Micela, G., Baliunas, S.L., Schmitt, J.H.M.M., Güdel, M., Harnden Jr, F.R., Sciortino, S., Stern, R.A., 2004, "High-amplitude, long-term X-ray variability in the solar-type star HD 81809: The beginning of an X-ray activity cycle?", Astron. Astrophys., 418, L13-L16. [DOI], [ADS] 4.5, 5

Living Reviews in Solar Physics

http://www. livingreviews . org/lrsp-2008-2 
Fawzy, D., Rammacher, W., Ulmschneider, P., Muielak, Z.E., Stępień, K., 2002, "Acoustic and magnetic wave heating in stars. I. Theoretical chromospheric models and emerging radiative fluxes", Astron. Astrophys., 386, 971-982. [DOI], [ADS] 4.4

Fleming, T.A., Giampapa, M.S., 1989, "A search for chromospheres at faint magnitudes", Astrophys. J., 346, 299-302. [DOI], [ADS] 2.3

Fleming, T.A., Giampapa, M.S., Garza, D., 2003, "The Quiescent Corona of VB 10", Astrophys. J., 594, 982-986. [DOI], [ADS] 2.3

Fontenla, J.M., Avrett, E.H., Loeser, R., 1993, "Energy Balance in the Solar Transition Region. III. Helium Emission in Hydrostatic, Constant-Abundance Models with Diffusion", Astrophys. $J .$, 406, 319-345. [DOI], [ADS] 3.4, 3.7

Fosbury, R.A.E., 1974, "The Source Functions of Some Strong Lines in Late-Type Stellar Atmospheres", Mon. Not. R. Astron. Soc., 169, 147-155. [ADS] 3.1

Fossum, A., Carlsson, M., 2005, "High-Frequency Acoustic Waves are Not Sufficient to Heat the Solar Chromosphere", Nature, 435, 919-921. [DOI], [ADS] 4.4

Fossum, A., Carlsson, M., 2006, "Determination of the Acoustic Wave Flux in the Lower Solar Chromosphere", Astrophys. J., 646, 579-592. [DOI], [ADS] 4.4

Foukal, P., Lean, J.L., 1990, "An Empirical Model of Total Solar Irradiance Variation Between 1874 and 1988", Science, 247, 556-558. [DOI], [ADS] 4.7

Foukal, P., Fröhlich, C., Spruit, H., Wigley, T.M.L., 2006, "Variations in solar luminosity and their effect on the Earth's climate", Nature, 443, 161-166. [DOI], [ADS] 4.9

Foukal, P.V., Vernazza, J.E., 1979, "The Effect of Magnetic Fields on Solar Luminosity", Astrophys. J., 234, 707-715. [DOI], [ADS] 4.7

Freire Ferrero, R., Gouttebroze, P., Catalano, S., Marilli, E., Bruhweiler, F., Kondo, Y., van der Hucht, K., Talavera, A., 1995, "Chromospheric Models for Altair (A7 IV-V)", Astrophys. J., 439, 1011-1020. [DOI], [ADS] 2.3

Frick, P., Baliunas, S.L., Galyagin, D., Sokoloff, D., Soon, W.H., 1997, "Wavelet Analysis of Stellar Chromospheric Activity Variations", Astrophys. J., 483, 426-434. [DOI], [ADS] 4.9

Friel, E., Cayrel de Strobel, G., Chmielewski, Y., Spite, M., Lèbre, A., Bentolila, C., 1993, "In search of real solar twins. III.", Astron. Astrophys., 274, 825-837. [ADS] 4

Friis-Christensen, E., Lassen, K., 1991, "Length of the Solar Cycle: An Indicator of Solar Activity Closely Associated with Climate", Science, 254, 698-700. [DOI], [ADS] 4.9

Friis-Christensen, E., Fröhlich, C., Haigh, J.D., Schüssler, N., von Steiger, R. (Eds.), 2000, Solar Variability and Climate, Proceedings of an ISSI Workshop, 28 June-2 July 1999, Bern, Switzerland, vol. 11 of Space Sciences Series, Kluwer, Dordrecht; Boston 4.9

Fröhlich, C., "PMOD Composite Total Solar Irradiance", project homepage, PMOD-WRC. URL (cited on 26 August 2007):

http://www.pmodwrc.ch/pmod.php?topic=tsi/composite/SolarConstant 4

Fröhlich, C., 1987, "Variability of the Solar 'Constant' on Time Scales of Minutes to Years", J. Geophys. Res., 92, 796-800. [DOI], [ADS] 4.7 
Fröhlich, C., Lean, J.L., 1998, "The Sun's Total Irradiance: Cycles, Trends, and Related Climate Change Uncertainties since 1976", Geophys. Res. Lett., 25, 4377-4380. [DOI], [ADS] 3.5, 4.7

Giampapa, M.S., Liebert, J., 1986, "High-Resolution Halpha Observations of M Dwarf Stars: Implications for Stellar Dynamo Models and Stellar Kinematic Properties at Faint Magnitudes", Astrophys. J., 305, 784-794. [DOI], [ADS] 2.3

Giampapa, M.S., Worden, S.P., Schneeberger, T.J., Cram, L.E., 1981, "Stellar Model Chromospheres. XII. High-Resolution, Absolute Flux Profiles of the Ca II H and K Lines in dMe and Non-dMe Stars", Astrophys. J., 246, 502-514. [DOI], [ADS] 3.4

Giampapa, M.S., Hall, J.C., Radick, R.R., Baliunas, S.L., 2006, "A Survey of Chromospheric Activity in the Solar-Type Stars in the Open Cluster M67", Astrophys. J., 651, 444-461. [DOI], [ADS] 4.1, 5

Gilliland, R.L., 1981, "Solar Radius Variations Over the Past 265 Years", Astrophys. J., 248, 1144-1155. [DOI], [ADS] 4.9

Goldberg, L., Noyes, R.W., Parkinson, W.H., Reeves, E.M., Withbroe, G.L., 1968, "Ultraviolet Solar Images from Space", Science, 162, 95-99. [DOI], [ADS] 4.5

Gray, R.O., Corbally, C.J., Garrison, R.F., McFadden, M.T., Robinson, P.E., 2003, "Contributions to the Nearby Stars (NStars) Project: Spectroscopy of Stars Earlier than M0 within 40 Parsecs: The Northern Sample. I", Astron. J., 126, 2048-2059. [DOI], [ADS] 4.1, 4.9

Gray, R.O., Corbally, C.J., Garrison, R.F., McFadden, M.T., Bubar, E.J., McGahee, C.E., O'Donoghue, A.A., Knox, E.R., 2006, "Contributions to the Nearby Stars (NStars) Project: Spectroscopy of Stars Earlier than M0 within 40 pc - The Southern Sample", Astron. J., 132, 161-170. [DOI], [ADS] 4.1, 11, 4.3, 12

Haigh, J.D., 2001, "Climate Variability and the Influence of the Sun", Science, 294, 2109-2111. [DOI] 4.9

Haigh, J.D., 2007, "The Sun and the Earth's Climate", Living Rev. Solar Phys., 4, lrsp-2007-2. URL (cited on 20 December 2007):

http://www.livingreviews.org/lrsp-2007-2 4.9

Haigh, J.D., Lockwood, M., Giampapa, M.S., 2005, The Sun, Solar Analogs, and the Climate, vol. 34 of Saas-Fee Advanced Courses, Springer, Berlin; New York. [Google Books]. Lecture notes of the 34th Advanced Course of the Swiss Society of Astronomy and Astrophysics in Davos, March 15-20, 2004, Eds. Rüedli, I. and Güdel, M. and Schmutz, W. 4.9

Hall, D.S., 1976, "The RS CVn Binaries and Binaries with Similar Properties", in Multiple Periodic Variable Stars, (Ed.) Fitch, W.S., Proceedings of IAU Colloquium 29, held in Budapest, Hungary, September 1-15, 1975, vol. 60 of Astrophysics and Space Science Library, p. 287, Reidel, Dordrecht; Boston. [ADS] 4.9

Hall, J.C., 1996, "On the Determination of Empirical Stellar Flux Scales", Publ. Astron. Soc. Pac., 108, 313-326. [DOI], [ADS] 4.2

Hall, J.C., Lockwood, G.W., 1995, "The Solar-Stellar Spectrograph: Project Description, Data Calibration and Initial Results", Astrophys. J., 438, 404-419. [DOI], [ADS] 4.9

Hall, J.C., Lockwood, G.W., 1998, "The Solar Activity Cycle. I. Observations of the End of Cycle 22, 1993 September-1997 February", Astrophys. J., 493, 494-504. [DOI], [ADS] 5

Living Reviews in Solar Physics

http://www . livingreviews . org/lrsp-2008-2 
Hall, J.C., Lockwood, G.W., 2004, "The Chromospheric Activity and Variability of Cycling and Flat Activity Solar-Analog Stars", Astrophys. J., 614, 942-946. [DOI], [ADS] 4.8

Hall, J.C., Henry, G.W., Lockwood, G.W., 2007a, "The Sun-Like Activity of the Solar Twin 18 Scorpii", Astron. J., 133, 2206-2208. [DOI], [ADS] 4.7, 16, 5

Hall, J.C., Lockwood, G.W., Skiff, B.A., 2007b, "The Activity and Variability of the Sun and Sun-Like Stars. I. Synoptic Ca II H and K Observations", Astron. J., 133, 862-881. [DOI], [ADS] $2.3,4.1,9,4.3,12,4.6,17,4.9,5$

Hardorp, J., 1978, "The Sun Among the Stars. I. A Search for Solar Spectral Analogs", Astron. Astrophys., 63, 383-390. [ADS] 4

Hartmann, L., Soderblom, D.R., Noyes, R.W., Burnham, N., Vaughan, A.H., 1984, "An Analysis of the Vaughan-Preston Survey of Chromospheric Emission", Astrophys. J., 276, 254-265. [DOI], [ADS] 4.3

Hathaway, D.H., Wilson, R.M., Reichmann, E.J., 1999, "A Synthesis of Solar Cycle Prediction Techniques", J. Geophys. Res., 104, 22,375-22,388. [DOI], [ADS] 4.9

Hayes, D.S., 1970, "An Absolute Spectrophotometric Calibration of the Energy Distribution of Twelve Standard Stars", Astrophys. J., 159, 165-176. [DOI], [ADS] 4.2

Hayes, D.S., Latham, D.W., 1975, "A Rediscussion of the Atmospheric Extinction and the Absolute Spectral-Energy Distribution of Vega", Astrophys. J., 197, 593-601. [DOI], [ADS] 4.2

Heasley, J.N., Ridgway, S.T., Carbon, D.F., Milkey, R.W., Hall, D.N.B., 1978, "The Fundamental Bands of CO in Arcturus: Evidence for an Inhomogeneous Chromosphere", Astrophys. J., 219, 970-978. [DOI], [ADS] 3.4

Hempelmann, A., 2003, "Wavelet analysis of stellar differential rotation. III. The Sun in white light", Astron. Astrophys., 399, 717-721. [DOI], [ADS] 4.1

Hempelmann, A., Schmitt, J.H.M.M., Stępień, K., 1996, "Coronal X-ray emission of cool stars in relation to chromospheric activity and magnetic cycles", Astron. Astrophys., 305, 284-295. [ADS] 4.5

Hempelmann, A., Robrade, J., Schmitt, J.H.M.M., Favata, F., Baliunas, S.L., Hall, J.C., 2006, "Coronal Activity Cycles in 61 Cygni", Astron. Astrophys., 460, 261-267. [DOI], [ADS] 4.5, 13, 5

Henry, G.W., 1999, "Techniques for Automated High-Precision Photometry of Sun-Like Stars", Publ. Astron. Soc. Pac., 111, 845-860. [DOI], [ADS] 4.7

Henry, T.J., Soderblom, D.R., Donahue, R.A., Baliunas, S.L., 1996, "A Survey of CaII H and K Chromospheric Emission in Southern Solar-Type Stars", Astron. J., 111, 439-465. [DOI], [ADS] $4.1,10,4.1,4.3,12$

Hilditch, R.W., 2001, An Introduction to Close Binary Stars, Cambridge University Press, Cambridge; New York. [Google Books] 4.9

Horne, J.H., Baliunas, S.L., 1986, "A Prescription for Period Analysis of Unevenly Sampled Time Series", Astrophys. J., 302, 757-763. [DOI], [ADS] 4.9

Hoyt, D.V., Schatten, K.H., 1997, The Role of the Sun in Climate Change, Oxford University Press, New York. [Google Books] 4.9 
Jefferies, J.T., Thomas, R.N., 1958, "The Source Function in a Non-Equilibrium Atmosphere. II. The Depth Dependence of the Source Function for Resonance and Strong Subordinate Lines", Astrophys. J., 127, 667-675. [DOI], [ADS] 3.7

Jefferies, J.T., Thomas, R.N., 1959, "Source Function in a Non-Equilibrium Atmosphere. III. The Influence of a Chromosphere", Astrophys. J., 129, 401-407. [DOI], [ADS] 3.1, 3.7

Jefferies, S.M., McIntosh, S.W., Armstrong, J.D., Bogdan, T.J., Cacciani, A., Fleck, B., 2006, "Magnetoacoustic Portals and the Basal Heating of the Solar Chromosphere", Astrophys. J. Lett., 648, L151-L155. [DOI], [ADS] 4.4

Jordan, C., Linsky, J.L., 1987, "Chromospheres and Transition Regions", in Exploring the Universe with the IUE Satellite, (Ed.) Kondo, Y., vol. 129 of Astrophysics and Space Science Library, pp. 259-293, D. Reidel, Dordrecht; Boston. [ADS] 3.3

Jordan, S.D., Avrett, E.H. (Eds.), 1973, Stellar Chromospheres, Proceedings of IAU Colloquium 19, held in Greenbelt, MD, 21 - 24 February, 1972, NASA, Washington. [ADS] 2.3, 2.4, 5

Joy, A.H., Abt, H.A., 1974, "Spectral Types of M Dwarf Stars", Astrophys. J. Suppl. Ser., 28, 1-18. [DOI], [ADS] 2.3

Judge, P.G., 1990, "On the Interpretation of Chromospheric Emission Lines", Astrophys. J., 348, 279-296. [DOI], [ADS] 4.9

Judge, P.G., Saar, S.H., 2007, "The Outer Solar Atmosphere During the Maunder Minimum: A Stellar Perspective", Astrophys. J., 663, 643-656. [DOI], [ADS] 4.8

Judge, P.G., Jordan, C., Rowan-Robinson, M., 1987, " $\delta$ Andromedae (K3 III): an IRAS source with an unusual ultraviolet spectrum", Mon. Not. R. Astron. Soc., 224, 93-106. [ADS] 2.3

Judge, P.G., Carlsson, M., Stein, R.F., 2003, "On the Origin of the Basal Emission from Stellar Atmospheres: Analysis of Solar C II Lines", Astrophys. J., 597, 1158-1177. [DOI], [ADS] 4.4

Judge, P.G., Saar, S.H., Carlsson, M., Ayres, T.R., 2004, "A Comparison of the Outer Atmosphere of the 'Flat Activity' Star $\tau$ Ceti (G8 V) with the Sun (G2 V) and $\alpha$ Centauri A (G2 V)", Astrophys. J., 609, 392-406. [DOI], [ADS] 4.8

Kalkofen, W., 2001, "The Case against Cold, Dark Chromospheres", Astrophys. J., 557, 376-383. [DOI], [ADS] 3.6

Kalkofen, W., Ulmschneider, P., Avrett, E.H., 1999, "Does the Sun Have a Full-Time Chromosphere?", Astrophys. J. Lett., 521, L141-L144. [DOI], [ADS] 3.6

Keil, S.L., Worden, S.P., 1984, "Variations in the Solar Calcium K Line 1976-1982", Astrophys. J., 276, 766-781. [DOI], [ADS] 3.2, 4.6

Kelch, W.L., 1978, "Stellar Model Chromospheres. VIII. 70 Ophiuchi A (K0 V) and $\epsilon$ Eridani (K2 V)", Astrophys. J., 222, 931-940. [DOI], [ADS] 3.4

Keller, C.U., Schüssler, M., Vögler, A., Zakharov, V., 2004, "On the Origin of Solar Faculae", Astrophys. J. Lett., 607, L59-L62. [DOI], [ADS] 3.5

Knaack, R., Fligge, M., Solanki, S.K., Unruh, Y.C., 2001, "The Influence of an Inclined Rotation Axis on Solar Irradiance", Astron. Astrophys., 376, 1080-1089. [DOI], [ADS] 4.7

Krivova, N.A., Balmaceda, L., Solanki, S.K., 2007, "Reconstruction of solar total irradiance since 1700 from the surface magnetic flux", Astron. Astrophys., 467, 335-346. [DOI], [ADS] 4.7, 4.8

Living Reviews in Solar Physics

http: //www. livingreviews.org/lrsp-2008-2 
Kuhn, J.R., Libbrecht, K.G., 1991, "Nonfacular Solar Luminosity Variations", Astrophys. J. Lett., 381, L53-L37. [DOI], [ADS] 4.7

Kuhn, J.R., Bush, R.I., Emilio, M., Scherrer, P.H., 2004, "On the Constancy of the Solar Diameter. II.", Astrophys. J., 613, 1241-1252. [DOI], [ADS] 4.9

Lean, J.L., Skumanich, A., White, O.R., 1992, "Estimating the Sun's Radiative Output During the Maunder Minimum", Geophys. Res. Lett., 19, 1591-1594. [DOI], [ADS] 4.8

Lean, J.L., Beer, J., Bradley, R., 1995, "Reconstruction of Solar Irradiance Since 1610: Implications for Climate Change", Geophys. Res. Lett., 22, 3195-3198. [DOI], [ADS] 4.8

Lean, J.L., Cook, J., Marquette, W., Johannesson, A., 1998, "Magnetic Sources of the Solar Irradiance Cycle", Astrophys. J., 492, 390-401. [DOI], [ADS] 4.7

Linsky, J.L., 1980, "Stellar Chromospheres", Annu. Rev. Astron. Astrophys., 18, 439-488. [DOI], [ADS] 2.4

Linsky, J.L., 1985, "Nonradiative Activity Across the H-R Diagram: Which Types of Stars are Solar-Like?", Solar Phys., 100, 333-362. [DOI], [ADS] 2.4

Linsky, J.L., Ayres, T.R., 1978, "Stellar model chromospheres. VI. Empirical estimates of the chromospheric radiative losses of late-type stars", Astrophys. J., 220, 619-628. [DOI], [ADS] 4.2

Linsky, J.L., Haisch, B.M., 1979, "Outer Atmospheres of Cool Stars. I. The Sharp Division into Solar-Type and Non-Solar-Type Stars", Astrophys. J. Lett., 229, L27-L32. [DOI], [ADS] 2.3, 3.4

Linsky, J.L., Worden, S.P., McClintock, W., Robertson, R.M., 1979, "Stellar model chromospheres. $\mathrm{X}$. High-resolution, absolute flux profiles of the CaII $\mathrm{H}$ and $\mathrm{K}$ lines in stars of spectral types F0-M2", Astrophys. J. Suppl. Ser., 41, 47-74. [DOI], [ADS] 4.2, 4.9

Lites, B.W., 2002, "Characterization of Magnetic Flux in the Quiet Sun", Astrophys. J., 573, 431-444. [DOI], [ADS] 4.4

Lites, B.W., Scharmer, G.B., Berger, T.E., Title, A.M., 2004, "Three-Dimensional Structure of the Active Region Photosphere as Revealed by High Angular Resolution", Solar Phys., 221, 65-84. [DOI], [ADS] 3.5

Livingston, W., Milkey, R., Slaughter, C., 1977, "Solar luminosity variation. I. Ci 5380 as a temperature indicator and a search for global oscillations", Astrophys. J., 211, 281-287. [DOI], [ADS] 3.2

Livingston, W.C., Holweger, H., 1982, "Solar Luminosity Variation. IV. The Photospheric Lines, 1976-1980", Astrophys. J., 252, 375-385. [DOI], [ADS] 3.2, 4.6, 5

Livingston, W.C., Wallace, L., White, O.R., Giampapa, M.S., 2006, "Sun-as-a-Star Spectrum Variations 1974-2006", Astrophys. J., 657, 1137-1149. [DOI], [ADS] 4.6, 14, 5

Lockwood, G.W., Skiff, B.A., Radick, R.R., 1997, "The Photometric Variability of Sun-like Stars: Observations and Results, 1984-1995", Astrophys. J., 485, 789-811. [DOI], [ADS] 4.7, 5

Lockwood, G.W., Skiff, B.A., Henry, G.W., Henry, S., Radick, R.R., Baliunas, S.L., Donahue, R.A., Soon, W.H., 2007, "Patterns of Photometric and Chromospheric Variation among Sunlike Stars: A 20 Year Perspective", Astrophys. J. Suppl. Ser., 171, 260-303. [DOI], [ADS] 4.7, 5 
Mauas, P.J.D., Falchi, A., Pasquini, L., Pallavicini, R., 1997, "Chromospheric Models of Dwarf M Stars", Astron. Astrophys., 326, 249-256. [ADS] 2.3

McClintock, W., Henry, R.C., Moos, H.W., Linsky, J.L., 1975, "Ultraviolet Observations of Cool Stars. IV. Intensities of Lyman- $\alpha$ and Mg II in epsilon Pegasi and epsilon Eridani, and Line Width-Luminosity Correlations", Astrophys. J., 202, 733-740. [DOI], [ADS] 3.2, 4.5

Meléndez, J., Ramírez, I., 2007, "HIP 56948: A Solar Twin with a Low Lithium Abundance", Astrophys. J. Lett., 669, L89-L92. [DOI], [ADS] 4.7, 5

Middelkoop, F., 1982, "Magnetic Structure in Cool Stars. IV. Rotation and Ca II H and K Emission of Main-sequence Stars", Astron. Astrophys., 107, 31-35. [ADS] 4.2

Middelkoop, F., Zwaan, C., 1981, "Magnetic Structure in Cool Stars. I. The Ca II H and K Emission from Giants", Astron. Astrophys., 101, 26-32. [ADS] 3.4

Mitchell Jr, W.E., Livingston, W.C., 1991, "Line-Blanketing Variations in the Irradiance Spectrum of the Sun From Maximum to Minimum of the Solar Cycle", Astrophys. J., 372, 336-348. [DOI], [ADS] 4.6

Montes, D., Fernández-Figueroa, M.J., De Castro, E., Cornide, M., Latorre, A., Sanz-Forcada, J., 2000, "Multiwavelength optical observations of ohromospherically active binary systems. III. High resolution echelle spectra from Ca II H \& K to Ca II IRT", Astron. Astrophys. Suppl., 146, 103-140. [DOI], [ADS] 4.9

Noël, F., 2004, "Solar cycle dependence of the apparent radius of the Sun", Astron. Astrophys., 413, 725-732. [DOI], [ADS] 4.9

Noyes, R.W., Hartmann, L.W., Baliunas, S.L., Duncan, D.K., Vaughan, A.H., 1984, "Rotation, Convection, and Magnetic Activity in Lower Main-Sequence Stars", Astrophys. J., 279, 763-777. [DOI], [ADS] 4.2, 4.3, 4.9

Oranje, B.J., 1986, "Magnetic structure in cool stars. IX. Ultraviolet emission lines from chromospheres and transition regions", Astron. Astrophys., 154, 185-196. [ADS] 3.3

Oranje, B.J., Zwaan, C., 1985, "Magnetic structure in cool stars. VIII. The Mg II h and k surface fluxes in relation to the Mt. Wilson photometric Ca II H and K measurements", Astron. Astrophys., 147, 265-272. [ADS] 3.3

Pace, G., Pasquini, L., Ortolani, S., 2003, "The Wilson-Bappu effect: A tool to determine stellar distances", Astron. Astrophys., 401, 997-1007. [DOI], [ADS] 3.2

Pap, J.M., Fox, P. (Eds.), 2003, Solar Variability and its Effects on Climate, vol. 141 of Geophysical Monograph, American Geophysical Union, Washington 4.9

Parker, E.N., 1955, "Hydromagnetic Dynamo Models", Astrophys. J., 122, 293-314. [DOI], [ADS] 3.5

Parker, E.N., 1975, "The Generation of Magnetic Fields in Astrophysical Bodies. X. Magnetic Buoyancy and the Solar Dynamo", Astrophys. J., 198, 205-209. [DOI], [ADS] 3.5

Pasquini, L., Pallavicini, R., 1991, "H $\alpha$ absolute chromospheric fluxes in G and K dwarfs and subgiants", Astron. Astrophys., 251, 199-209. [ADS] 4.2

Pasquini, L., Pallavicini, R., Pakull, M., 1988, "Ca II absolute line profiles of southern late-type stars", Astron. Astrophys., 191, 253-266. [ADS] 4.2

Living Reviews in Solar Physics

http: //www. livingreviews . org/lrsp-2008-2 
Penn, M.J., Livingston, W.C., 2006, "Temporal Changes in Sunspot Umbral Magnetic Fields and Temperatures", Astrophys. J. Lett., 649, L45-L48. [DOI], [ADS] 4.9

Porto de Mello, G.F., da Silva, L., 1997, "HR 6060: The Closest Ever Solar Twin?", Astrophys. J. Lett., 482, L89-L92. [DOI], [ADS] 4.7

Radick, R.R., Lockwood, G.W., Skiff, B.A., Baliunas, S.L., 1998, "Patterns of Variation Among Sun-like Stars", Astrophys. J. Suppl. Ser., 118, 239-258. [DOI], [ADS] 4.7, 4.7, 15, 5

Reimers, D., 1982, "Detection of Further Red Giants with 'Hybrid' Atmospheres and a Possible Correlation with Double Circumstellar Mg II and Ca II Lines", Astron. Astrophys., 107, 292-299. [ADS] 2.3

Reimers, D., Hünsch, M., Schmitt, J.H.M.M., Toussaint, F., 1996, "Hybrid stars and the reality of 'dividing lines' among G to K bright giants and supergiants", Astron. Astrophys., 310, 813-824. [ADS] 2.3

Reiners, A., Basri, G., 2007, "The First Direct Measurements of Surface Magnetic Fields on Very Low Mass Stars", Astrophys. J., 656, 1121-1135. [DOI], [ADS] 2.3

Ribes, J.C., Nesme-Ribes, E., 1993, "The solar sunspot cycle in the Maunder minimum AD1645 to AD1715", Astron. Astrophys., 276, 549-563. [ADS] 4.8

Rind, D., 2002, "The Sun's Role in Climate Variations", Science, 296, 673-677. [DOI], [ADS] 4.9

Roberts, W.O., 1945, "A Preliminary Report on Chromospheric Spicules of Extremely Short Lifetime", Astrophys. J., 101, 136-142. [DOI], [ADS] 2.1, 2

Rosner, R., Musielak, Z.E., Cattaneo, F., Moore, R.L., Suess, S.T., 1995, "On the Origin of 'Dividing Lines' for Late-Type Giants and Supergiants", Astrophys. J. Lett., 442, L25-L28. [DOI], [ADS] 2.3

Rutten, R.G.M., 1984, "Magnetic structure in cool stars. VII. Absolute surface flux in Ca II H and K line cores", Astron. Astrophys., 130, 353-360. [ADS] 4.9

Rutten, R.G.M., Schrijver, C.J., 1987, "Magnetic structure in cool stars. XIII. Appropriate units for the rotation-activity relation", Astron. Astrophys., 177, 155-162. [ADS] 4.2

Rutten, R.J., 2007a, "Observing the Solar Chromosphere", in The Physics of Chromospheric Plasmas, (Eds.) Heinzel, P., Dorotovic, I., Rutten, R.J, NSO Workshop No. 23, vol. 368 of ASP Conference Series, pp. 27-48, Astronomical Society of the Pacific, San Francisco 3.5

Rutten, R.J., 2007b, "On the Nature of the Solar Chromosphere", in Solar MHD Theory and Observations: A High Spatial Resolution Perspective, (Eds.) Leibacher, J., Stein, R.F., Uitenbrock, H., Proceedings of the conference held 18-22 July, 2005, at the National Solar Observatory, Sacramento Peak, Sunspot, New Mexico, USA, vol. 354 of ASP Conference Series, pp. 276-283, Astronomical Society of the Pacific, San Francisco 3.6

Saar, S.H., Brandenburg, A., 1999, "Time Evolution of the Magnetic Activity Cycle Period. II. Results for an Expanded Stellar Sample", Astrophys. J., 524, 295-310. [DOI], [ADS] 4.3, 5

Schatten, K., 2005, "Fair space weather for solar cycle 24", Geophys. Res. Lett., 32, L21 106. [DOI], [ADS] 4.9

Schrijver, C.J., 1987, "Magnetic structure in cool stars. XI. Relations between radiative fluxes measuring stellar activity, and evidence for two components in stellar chromospheres", Astron. Astrophys., 172, 111-123. [ADS] 3.3, 4.4 
Schrijver, C.J., 1992, "The basal and strong-field components of the solar atmosphere", Astron. Astrophys., 258, 507-520. [ADS] 4.4

Schrijver, C.J., 1995, "Basal heating in the atmospheres of cool stars: Observational evidence and theoretical support", Astron. Astrophys. Rev., 6, 181-223. [ADS] 4.4

Schrijver, C.J., Title, A.M., 2003, "The Magnetic Connection between the Solar Photosphere and the Corona", Astrophys. J. Lett., 597, L165-L168. [DOI], [ADS] 4.4

Schrijver, C.J., Coté, J., Zwaan, C., Saar, S.H., 1989a, "Relations Between the Photospheric Magnetic Field and the Emission from the Outer Atmospheres of Cool Stars. I. The Solar Ca II K Line Core Emission", Astrophys. J., 337, 964-976. [DOI], [ADS] 4.9

Schrijver, C.J., Dobson, A.K., Radick, R.R., 1989b, "The Magnetic, Basal, and RadiativeEquilibrium Components in Mount Wilson Ca II H + K Fluxes", Astrophys. J., 341, 1035-1044. [DOI], [ADS] 4.4, 4.9

Schrijver, C.J., Title, A.M., Berger, T.E., Fletcher, L., Hurlburt, N.E., Nightingale, R.W., Shine, R.A., Tarbell, T.D., Wolfson, J., Golub, L., Bookbinder, J.A., DeLuca, E.E., McMullen, R.A., Warren, H.P., Kankelborg, C.C., Handy, B.N., de Pontieu, B., 1999, "A New View of the Solar Outer Atmosphere by the Transition Region and Coronal Explorer", Solar Phys., 187, 261-302. [DOI], [ADS] 3.5, 3.6

Schüssler, M., Shelyag, S., Berdyugina, S., Vögler, A., Solanki, S.K., 2003, "Why solar magnetic flux concentrations are bright in molecular bands", Astrophys. J. Lett., 597, L173-L176. [DOI], [ADS] 5

Schwarzschild, M., 1948, "On Noise Arising from the Solar Granulation", Astrophys. J., 107, 1-5. [DOI], [ADS] 2.2

Schwarzschild, M., 1958, Structure and Evolution of the Stars, Princeton University Press, Princeton 3.1

Sello, S., 2003, "Solar cycle activity: A Prelimary Prediction for Cycle \#24", Astron. Astrophys. 410, 691-693. [DOI], [ADS] 4.9

Sheeley Jr, N.R., 1967, "The Average Profile of the Solar K-Line During the Sunspot Cycle", Astrophys. J., 147, 1106-1115. [ADS] 3.2, 3.7

Simon, T., 1986, "Stellar Chromospheres, Coronae, and Winds", Astrophys. Space Sci., 118, 209222. [DOI], [ADS] 2.4

Simon, T., Landsman, W., 1991, "The Onset of Chromospheric Activity Among the A and F Stars", Astrophys. J., 380, 200-207. [DOI], [ADS] 2.3

Simon, T., Ayres, T.R., Redfield, S., Linsky, J.L., 2002, "Limits on Chromospheres and Convection Among the Main-Sequence A Stars", Astrophys. J., 579, 800-809. [DOI], [ADS] 2.3

Skumanich, A., 1970, "On the Existence of a Purely Radiative Temperature Rise in the Solar Chromosphere", Astrophys. J., 159, 1077-1078. [DOI], [ADS] 2.2

Skumanich, A., 1972, "Time Scales for Ca II Emission Decay, Rotational Braking, and Lithium Depletion", Astrophys. J., 171, 565-567. [DOI], [ADS] 3.2

Soderblom, D.R., Duncan, D.K., Johnson, D.R.H., 1991, "The Chromospheric Emission-Age Relation for Stars of the Lower Main Sequence and its Implications for the Star Formation Rate", Astrophys. J., 375, 722-739. [DOI], [ADS] 3.7 
Solanki, S.K., Livingston, W.C., Ayres, T.R., 1994, "New Light on the Heart of Darkness of the Solar Chromosphere", Science, 263, 64-66. [DOI], [ADS] 2.1

Solanki, S.K., Usoskin, I.G., Kromer, B., Schüssler, M., Beer, J., 2004, "Unusual activity of the Sun during recent decades compared to the previous 11,000 years", Nature, 431, 1084-1087. [DOI], [ADS] 4.9

Soon, W.H., Baliunas, S.L., Zhang, Q., 1993, "An Interpretation of Cycle Periods of Stellar Chromospheric Activity", Astrophys. J. Lett., 414, L33-L36. [DOI], [ADS] 4.3

Soubiran, C., Triaud, A., 2004, "The Top Ten Solar Analogs in the ELODIE Library", Astron. Astrophys., 418, 1089-1100. [DOI], [ADS] 4, 5

Spruit, H.C., 1977, "Heat flow near obstacles in the solar convection zone", Solar Phys., 55, 3-34. [DOI], [ADS] 3.5

Spruit, H.C., Roberts, B., 1983, "Magnetic flux tubes on the Sun", Nature, 304, 401-406. [DOI] 3.5

Strassmeier, K.G., 1996, "Doppler imaging of stellar surface structure. I. The rapidly-rotating RS CVn binary UZ Librae", Astron. Astrophys., 314, 558-566. [ADS] 4.1, 4.9

Strassmeier, K.G., 2005, "Stellar Activity Cycles: Observing the Dynamo?", Astron. Nachr., 326, 269-277. [DOI], [ADS] 3.7

Strassmeier, K.G., Hall, D.S., Fekel, F.C., Scheck, M., 1993, "A catalog of chromospherically active binary stars (second edition)", Astron. Astrophys. Suppl., 100, 173-225. [ADS]. Related online version (cited on 31 January 2008):

http://cdsarc.u-strasbg.fr/viz-bin/Cat?V/76= 4.9

Suematsu, Y., Wang, H., Zirin, H., 1995, "High-Resolution Observation of Disk Spicules. I. Evolution and Kinematics of Spicules in the Enhanced Network", Astrophys. J., 450, 411-421. [DOI], [ADS] 3.5

Thomas, R.N., 1957, "The Source Function in a Non-Equilibrium Atmosphere. I. The Resonance Lines", Astrophys. J., 125, 260-274. [DOI], [ADS] 3.1, 3.7

Tüg, H., White, N.M., Lockwood, G.W., 1977, "Absolute Energy Distribution of $\alpha$ Lyrae and 109 Virginis from $3295 \AA$ to $9040 \AA$ ", Astron. Astrophys., 61, 679-684. [ADS] 4.2

Uitenbroek, H., 2000, "The CO Fundamental Vibration-Rotation Lines in the Solar Spectrum. II. Non-LTE Transfer Modeling in Static and Dynamic Atmospheres", Astrophys. J., 536, 481-493. [DOI], [ADS] 3.4

Ulmschneider, P., 1979, "Stellar Chromospheres", Space Sci. Rev., 24, 71-100. [DOI], [ADS] 2.4

Ulmschneider, P., Kalkofen, W., Nowak, T., Bohn, U., 1977, "Acoustic Waves in the Solar Atmosphere I, The Hydrodynamic Code", Astron. Astrophys., 54, 61-70. [ADS] 2.4

Unruh, Y.C., Solanki, S.K., Fligge, M., 1999, "The Spectral Dependence of Facular Contrast and Solar Irradiance Variations", Astron. Astrophys., 345, 635-642. [ADS] 4.6

Usoskin, I.G., Solanki, S.K., Kovaltsov, G.A., 2007, "Grand minima and maxima of solar activity: new observational constraints", Astron. Astrophys., 471, 301-309. [DOI], [ADS], [arXiv:0706.0385] 5 
Vaughan, A.H., 1980, "Comparison of Activity Cycles in Old and Young Main-Sequence Stars", Publ. Astron. Soc. Pac., 92, 392-396. [DOI], [ADS] 4.3

Vaughan, A.H., Preston, G.W., 1980, "A Survey of Chromospheric Ca II H and K Emission in Field Stars of the Solar Neighborhood", Publ. Astron. Soc. Pac., 92, 385-391. [DOI], [ADS] 4.3, 12

Vaughan, A.H., Preston, G.W., Wilson, O.C., 1978, "Flux Measurements of Ca II H and K Emission", Publ. Astron. Soc. Pac., 90, 267-274. [DOI], [ADS] 4.2

Vernazza, J.E., Avrett, E.H., Loeser, R., 1973, "Structure of the Solar Chromosphere. I. Basic Computations and Summary of the Results", Astrophys. J., 184, 605-631. [DOI], [ADS] 3.1

Vernazza, J.E., Avrett, E.H., Loeser, R., 1981, "Structure of the Solar Chromosphere. III. Models of the EUV Brightness Components of the Quiet Sun", Astrophys. J. Suppl. Ser., 45, 635-725. [DOI], [ADS] 2.1, 3, 3.1, 3.7

Vogt, S.S., Penrod, G.D., Hatzes, A.P., 1987, "Doppler Images of Rotating Stars Using Maximum Entropy Image Reconstruction", Astrophys. J., 321, 496-515. [DOI], [ADS] 4.9

Wang, Y.-M., Lean, J.L., Sheeley Jr, N.R., 2005, "Modeling the Sun's Magnetic Field and Irradiance since 1713", Astrophys. J., 625, 522-538. [DOI], [ADS] 4.8, 4.9

Weiler, E.J., Oegerle, W.R., 1979, "A Copernicus Survey of Mg II Emission in Late-Type Stars", Astrophys. J. Suppl. Ser., 39, 537-547. [DOI], [ADS] 3.2

White, O.R., Livingston, W.C., 1978, "Solar Luminosity Variation. II. Behavior of Calcium H and $\mathrm{K}$ at Solar Minimum and the Onset of Cycle 21", Astrophys. J., 226, 679-686. [DOI], [ADS] 3.2

White, O.R., Livingston, W.C., 1981, "Solar Luminosity Variation. III. Calcium K Variation from Solar Minimum to Maximum in Cycle 21", Astrophys. J., 249, 798-816. [DOI], [ADS] 3.2, 4.6

White, O.R., Livingston, W.C., Wallace, L., 1987, "Variability of Chromospheric and Photospheric Lines in Solar Cycle 21", J. Geophys. Res., 92, 823-827. [DOI], [ADS] 3.2

White, O.R., Skumanich, A., Lean, J.L., Livingston, W.C., Keil, S.L., 1992, "The Sun in a Noncycling State", Publ. Astron. Soc. Pac., 104, 1139-1132. [DOI], [ADS] 4.8

Wiedemann, G., Ayres, T.R., Jennings, D.E., Saar, S.H., 1994, "Carbon Monoxide Fundamental Bands in Late-Type Stars. III. Chromosphere or CO-mosphere?", Astrophys. J., 423, 806-816. [DOI], [ADS] 3.6

Willson, R.C., 1997, "Total Solar Irradiance Trend During Solar Cycles 21 and 22", Science, 277, 1963-1965. [DOI], [ADS] 4.7

Wilson, O.C., 1963, "A Probable Correlation Between Chromospheric Activity and Age in MainSequence Stars", Astrophys. J., 138, 832-848. [DOI], [ADS] 3.2, 3.7

Wilson, O.C., 1968, "Flux Measurements at the Centers of Stellar H and K Lines", Astrophys. J., 153, 221-234. [DOI], [ADS] 3.7

Wilson, O.C., 1978, "Chromospheric Variations in Main-Sequence Stars", Astrophys. J., 226, 379396. [DOI], [ADS] 3.2, 4.1, 4.2

Wilson, O.C., Bappu, M.K.V., 1957, "H and K Emission in Late-Type Stars: Dependence of Line Width on Luminosity and Related Topics", Astrophys. J., 125, 661-684. [DOI], [ADS] 3.2

Living Reviews in Solar Physics

http://www. livingreviews.org/lrsp-2008-2 
Wilson, O.C., Skumanich, A., 1964, "Dependence of Chromospheric Activity upon Age in MainSequence Stars: Additional Evidence", Astrophys. J., 140, 1401-1408. [DOI], [ADS] 3.2, 3.7

Worden, J.R., White, O.R., Woods, T.N., 1998, "Evolution of Chromospheric Structures Derived from Ca II K Spectroheliograms: Implications for Solar Ultraviolet Irradiance Variability", Astrophys. J., 496, 998-1014. [DOI], [ADS] 4.6

Wright, J.T., 2004, "Do We Know of any Maunder Minimum Stars?", Astron. J., 128, 1273-1278. [DOI] 4.8

Wright, J.T., Marcy, G.W., Butler, R.P., Vogt, S.S., 2004, "Chromospheric Ca II Emission in Nearby F, G, K, and M Stars", Astrophys. J. Suppl. Ser., 152, 261-295. [DOI], [ADS] 3.7, 4.1, 4.9

Zhang, Q., Soon, W.H., Baliunas, S.L., Lockwood, G.W., Skiff, B.A., Radick, R.R., 1994, "A Method for Determining Possible Brightness Variations of the Sun in Past Centuries from Observations of Solar-Type Stars", Astrophys. J. Lett., 427, L111-L114. [DOI], [ADS] 4.8 